ARTICLE

\title{
Regulatory protein HilD stimulates Salmonella Typhimurium invasiveness by promoting smooth swimming via the methyl-accepting chemotaxis protein $\mathrm{McpC}$
}

Kendal G. Cooper ${ }^{1}$, Audrey Chong ${ }^{1}$, Laszlo Kari ${ }^{1}$, Brendan Jeffrey ${ }^{2}$, Tregei Starr ${ }^{1,7}$, Craig Martens ${ }^{3}$, Molly McClurg ${ }^{4}$, Victoria R. Posada ${ }^{4}$, Richard C. Laughlin ${ }^{4}$, Canaan Whitfield-Cargile (D) ${ }^{5}$, L. Garry Adams (D) ${ }^{6}$, Laura K. Bryan (10 6 , Sara V. Little (iD) ${ }^{6}$, Mary Krath (i) ${ }^{6}$, Sara D. Lawhon ${ }^{6} \&$ Olivia Steele-Mortimer (i) ${ }^{1 凶}$

In the enteric pathogen Salmonella enterica serovar Typhimurium, invasion and motility are coordinated by the master regulator HilD, which induces expression of the type III secretion system 1 (T3SS1) and motility genes. Methyl-accepting chemotaxis proteins (MCPs) detect specific ligands and control the direction of the flagellar motor, promoting tumbling and changes in direction (if a repellent is detected) or smooth swimming (in the presence of an attractant). Here, we show that HilD induces smooth swimming by upregulating an uncharacterized MCP (McpC), and this is important for invasion of epithelial cells. Remarkably, in vitro assays show that $\mathrm{McpC}$ can suppress tumbling and increase smooth swimming in the absence of exogenous ligands. Expression of $m c p C$ is repressed by the universal regulator $\mathrm{H}-\mathrm{NS}$, which can be displaced by HilD. Our results highlight the importance of smooth swimming for Salmonella Typhimurium invasiveness and indicate that McpC can act via a ligand-independent mechanism when incorporated into the chemotactic receptor array.

\footnotetext{
${ }^{1}$ Laboratory of Bacteriology, Rocky Mountain Laboratory, National Institutes of Allergy and Infectious Diseases, National Institutes of Health, Hamilton, MT 59840, USA. ${ }^{2}$ NIAID Bioinformatics and Computational Biosciences Branch, Rocky Mountain Laboratory, National Institutes of Allergy and Infectious Diseases, National Institutes of Health, Hamilton, MT 59840, USA. ${ }^{3}$ NIAID RML Research Technologies Section, Genomics Unit, Rocky Mountain Laboratory, National Institutes of Allergy and Infectious Diseases, National Institutes of Health, Hamilton, MT 59840, USA. ${ }^{4}$ Department of Biological and Health Sciences, Texas A\&M University-Kingsville, Kingsville, TX 78363, USA. ${ }^{5}$ Department of Veterinary Large Animal Clinical Sciences, Texas A\&M College of Veterinary Medicine and Biomedical Sciences, College Station, TX 77843, USA. ${ }^{6}$ Department of Veterinary Pathobiology, Texas A\&M College of Veterinary Medicine and Biomedical Sciences, College Station, TX 77843, USA. ${ }^{7}$ Present address: GlaxoSmithKline, Hamilton, MT 59840, USA.

凶email: omortimer@niaid.nih.gov
} 
G enetically diverse groups of pathogenic microorganisms rely upon directed motility, i.e. chemotaxis, to optimally colonize host tissues. Chemotaxis allows motile bacterial cells to navigate through complex environments, such as the mammalian gastrointestinal tract. In the model organisms Escherichia coli and Salmonella, bacteria swim in a random pattern produced by alternating counterclockwise (CCW) and clockwise (CW) flagellar rotation. Chemoreceptors detect attractants or repellents and stimulate responses through a signaling cascade that controls the direction of the flagellar motor. Attractant gradients extend the length of time flagellar motors rotate CCW, resulting in more smooth swimming in a favorable direction, while repellents cause an increase of $\mathrm{CW}$ rotations, resulting in more tumbling and changes in direction. Chemotaxis is required for Salmonella enterica serovar Typhimurium (STm) growth in the lumen of the inflamed gut ${ }^{1,2}$, however, nonchemotactic smooth swimming mutants are more invasive $\mathrm{e}^{3-5}$ suggesting that repression of chemotaxis could be advantageous under certain conditions.

Salmonella enterica serovars, including STm, are gut-adapted Gram-negative bacteria that cause disease in a wide variety of vertebrate species. In humans, STm typically causes a self-limiting diarrhea although it can cause severe systemic disease in immunecompromised individuals. Following ingestion, the bacteria colonize the small intestine, a step that is facilitated by the Salmonella pathogenicity island 1 (SPI1) and flagellar motility 6,7 . SPI1 encodes a type III secretion system (T3SS1), required for invasion of intestinal epithelial cells and gut inflammation, as well as several transcriptional regulators ${ }^{8}$. The SPI1 regulon is induced in the gut ${ }^{9-11}$, via mechanisms that are not fully understood, or under certain in vitro growth conditions ${ }^{12-14}$, which has enabled characterization of many of the players and revealed transcriptional cross talk with motility genes ${ }^{15-24}$. The SPI1-encoded transcription factors HilA and HilD are the dominant regulators of SPI1 expression. HilD activates $\mathrm{HilA}^{16,25}$ and also directly activates transcription of the flagellar master operon $f l h D C$ by binding upstream of the P5 promoter ${ }^{21}$. In fact, many flagellar proteins also target HilA or HilD ${ }^{14,26}$. Both SPI1 and flagellar genes exhibit bistable expression, i.e, expressing and nonexpressing bacteria coexist in the same populations.

In this work, we examine the role of chemotaxis in STm interactions with the intestinal epithelium. We hypothesize that invasion-primed (SPI1-induced) STm may preferentially navigate towards the gut epithelium. By investigating the HilD regulon and focusing on both the ability to invade epithelial cells and motility, we identify the methyl-accepting chemotaxis protein (MCP), $\mathrm{McpC}$, as a HilD-regulated protein that induces smooth swimming in T3SS1-expressing bacteria thus enhancing their net movement toward the mucosal epithelium. Our findings reveal a mechanism of directed motility that optimizes colonization of the gastrointestinal tract by Salmonella.

\section{Results}

In SPI1-induced STm, HilD increases internalization into host cells. To determine if the HilD regulon can control motility towards host cells in SPI1-induced bacteria, we performed internalization experiments using macrophages, which can take up Salmonella via both T3SS1-dependent and T3SS1-independent mechanisms. Since the contribution of chemotaxis and/or motility in invasion can be subtle, we compared a hilD deletion mutant $(\Delta h i l D)$ to a strain lacking the entire SPI1 $(\Delta$ SPI1), which also lacks $h i l D$, and the wild-type (WT) strain. As expected, since HilD is required for T3SS1 expression, mutants were internalized into human monocyte-derived macrophages (HuMDM) at a much lower level than WT (Fig. 1a). We next looked at a series of mutants that express hilD but are defective in T3SS1 expression $(\Delta h i l A)$ or function $(\Delta i n v A, \Delta s i p B$, and $\Delta p r g I)$. All of these mutants were internalized slightly better than the $\Delta$ SPI1 and $\Delta$ hilD strains, although without statistical significance. Nevertheless, episomal expression of hilD in the $\Delta$ SPI1 strain under the control of an arabinose inducible promoter $(\Delta \mathrm{SPI} 1 \mathrm{pBAD}-h i l D)$ resulted in increased internalization compared to a control strain ( $\triangle$ SPI1 pBAD-null) (Fig. 1b, left). A similar effect was seen in C2Bbe1 epithelial cells, into which low levels of T3SS1independent uptake also occurs (Fig. 1b, right). Thus, in SPI1induced STm, expression of hilD increases both T3SS1-dependent and T3SS1-independent internalization of STm into macrophages and epithelial cells.

RNAseq reveals that mcpC is a target of HilD. We used RNA sequencing to compare the motility transcriptome in a HilD overexpressing strain compared to WT under SPI1-inducing conditions. HilD ${ }^{+}$(hilD $\Delta 3^{\prime} \mathrm{UTR}$ ) was constructed by removing the hilD $3^{\prime}$-untranslated region ${ }^{27}$. As expected, expression of T3SS1 genes and other previously identified HilD targets were upregulated in $\mathrm{HilD}^{+28,29}$ (Table 1). RNAseq results can be found in Supplementary Data 1. Several motility genes were elevated in $\mathrm{HilD}^{+}$, however, only $m c p C$, which encodes for an MCP present in all Salmonella, but not in E. coli ${ }^{30}$, and previously identified as a potential member of the HilD regulon ${ }^{28,29}$ was significantly upregulated (Table 1).

The chemoreceptor McpC contributes to invasion. Coregulation of $m c p C$ with the T3SS1 suggests a role for this chemoreceptor in motility toward host cells, which could account for the ability of hilD to increase internalization of the $\triangle$ SPI1 mutant (Fig. 1b). Indeed, episomally expressed hilD did not rescue internalization of a $\Delta \mathrm{SPI} 1 / \Delta m c p C$ mutant (Fig. 1c), confirming that $m c p C$ is required for T3SS1-independent internalization under these conditions. McpC also plays a role in T3SS1dependent invasion, since $\triangle m c p C$ had a pronounced invasion defect in HeLa and C2Bbel cells that was rescued by episomal expression of $m c p C$ under its native promoter (Fig. 1d). Together these data suggest that $m c p C$ contributes to invasion efficiency.

HilD regulates mcpC by derepression of $\mathbf{H}-\mathrm{NS}$. Flagellar gene expression begins with the master operon $f h D C$ that is controlled by a class I promoter. $\mathrm{FlhD}_{4} \mathrm{C}_{2}$ promotes transcription of class II promoters of flagellar assembly genes and the flagellar-specific sigma factor fliA $(\sigma 28)$, which directs transcription of class III genes such as flagellin and chemoreceptors ${ }^{31}$. To determine whether $m c p C$ is a typical class III flagellar gene, and how HilD contributes to its expression, we monitored $m c p C$ promoter activity using a plasmid-based transcriptional GFP reporter. $\mathrm{P} m c p C-387 g f p$ contains the $387 \mathrm{bp}$ intergenic region between the stop codon of the gene aer and the translational start of $m c p C$ (Fig. 2a). Expression of $\mathrm{P} m c p C-387 g f p$ was assessed and compared in multiple strains (WT, $\Delta f l h D, \Delta f l i A, \Delta h i l D, \mathrm{HilD}^{+}$). Strikingly, this assay confirmed the dependence of $m c p C$ expression on HilD because GFP fluorescence was virtually undetectable in the $\Delta$ hilD background and was increased, compared to WT, in the $\mathrm{HilD}^{+}$background. FliA was also required for $m c p C$ expression (Fig. 2c). Thus, while $m c p C$ like other chemoreceptor genes, is a class III flagellar gene, it is unique in having an additional level of regulatory control that couples its expression to SPI1 induction via HilD.

To further characterize the role of HilD in the regulation of $m c p C$, we constructed a $5^{\prime}$ truncated reporter $(\mathrm{P} m c p C-79 g f p)$ that lacks the predicted HilD-binding site (Fig. 2b). Reporter expression was independent of HilD but still dependent on FliA 

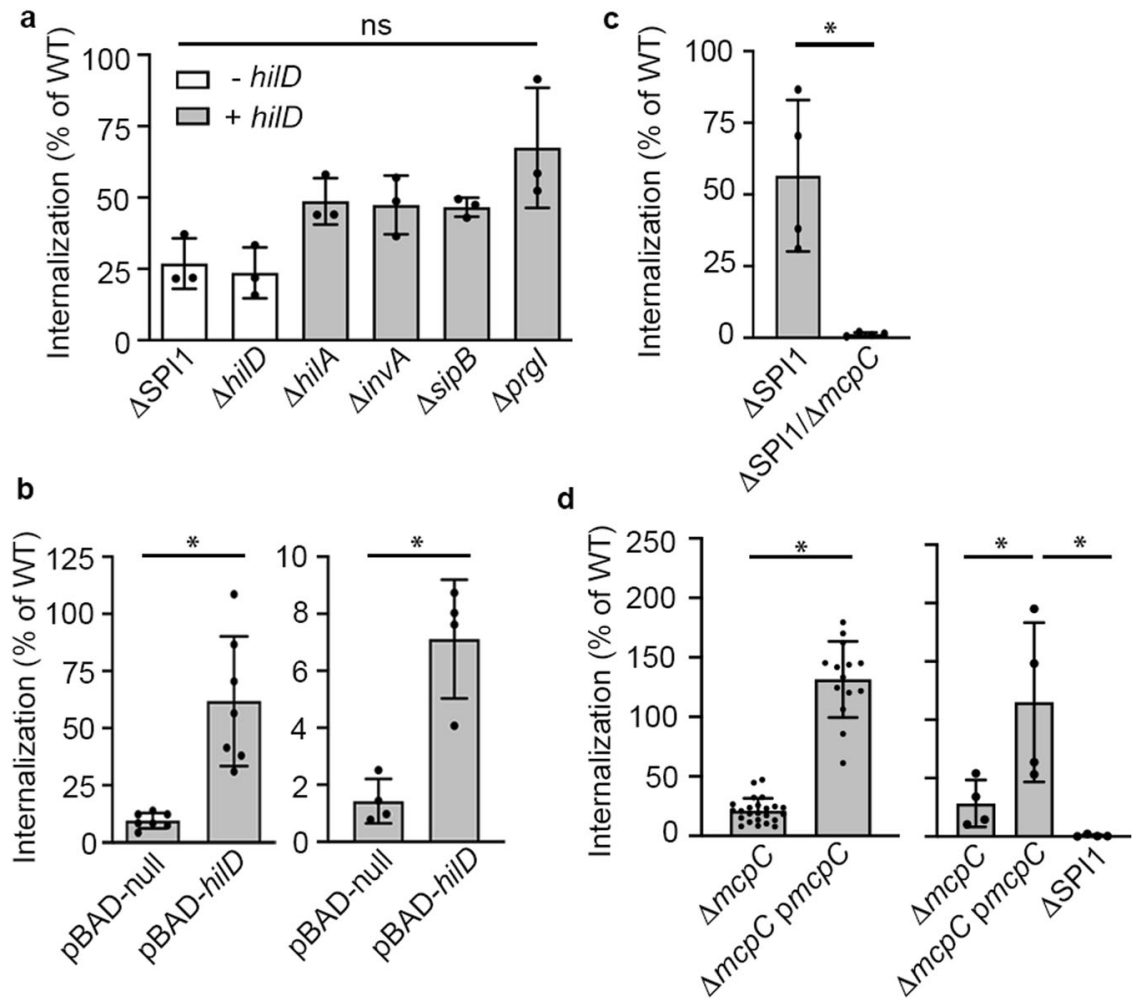

Fig. 1 HilD has contributions to invasion efficiency dependent on $\mathbf{m c p C}$. a Internalization (defined as the \% inoculum) at $2 \mathrm{~h}$ post-infection (pi) in human monocyte-derived macrophages (HuMDM). $n=3$, independent experiments with the mean \pm SD shown. + or - hilD indicates the presence or absence of hilD, respectively. $\mathbf{b}$ Internalization data of HuMDM (2 h pi, left) or C2Bbe1 (1.5 h pi, right) infected with $\Delta$ SPI1 induced for null or hilD expression. The mean \pm SD are shown from $n=7$ independent donors (HuMDM) or $n=4$ independent experiments (C2Bbe1). Statistical significances were determined using one-way Anova followed by Tukey's multiple comparisons. In HuMDM, $\triangle$ SPI1 pBAD-null vs. $\triangle$ SPI1 pBAD-hilD: $p=0.005$, in C2Bbe1, $\triangle$ SPI1 pBADnull vs. $\triangle$ SPI1 pBAD-hilD: $p=0.02$. c Internalization at $2 \mathrm{~h}$ pi of HuMDM comparing the $\triangle$ SPI1 mutant vs. $\Delta$ SPI1/ $\Delta m c p C$, each containing pBAD-hilD and induced for hilD expression. The mean \pm SD of $n=4$ independent experiments is shown. Statistical significance was determined using one-way Anova followed by Tukey's multiple comparisons ( $\Delta$ SPI1 pBAD-hilD vs. $\Delta$ SPI1/ $\Delta m p c C$ pBAD-hilD: $p=0.045$ ). d Internalization at $1.5 \mathrm{~h}$ pi into HeLa cells (left) or C2Bbe1 (right) of the indicated strains. The mean \pm SD in HeLa cells are shown from $n=24$ independent experiments comparing WT and $\Delta m p c C$ and $n=$ 14 of these included $\triangle m p c C$ pmpcC. Statistical significances were determined by one-way Anova with a mixed effects model if a value was missing, followed by Tukey's multiple comparisons (WT vs. $\Delta m p c C: p=0.0001, \Delta m p c C$ vs. $\Delta m p c C$ pmpcC: $p=0.0001$, WT vs. $\Delta m p c C$ pmpcC: $p=0.003$ ). The mean \pm SD in C2Bbe1 cells are shown from $n=4$ independent experiments. Statistical significances were determined by one-way Anova followed by Tukey's multiple comparisons ( $\triangle m p c C$ vs. $\Delta m p c C$ pmpcC: $p=0.02, \Delta$ SPI1 vs. $\Delta m p c C$ pmpcC: $p=0.004$ ). Source data are provided as a Source Data file. Growth curves for these strains are included in the Supplementary Information. ns = not significant, ${ }^{\star} p<0.05$.

(Fig. 2d). Levels of HilD-independent reporter expression were lower than that in the WT strain probably due to the loss of HilDpositive effects on flagellar gene expression including fliA, through interactions with the flhDC P5 promoter $^{21}$. In addition, $m c p C$ reporter expression in the hilD $D^{+}$strain was increased only in the presence of the HilD-binding site (compare WT and HilD ${ }^{+}$ strains in Fig. 2c, d). These data indicate that the region between -79 to -387 , which contains the HilD-binding site, has a repressing effect on $m c p C$ expression and support a derepression model by which HilD binding displaces a repressor of $m c p C$ expression, i.e. if the upstream region is removed, then HilD binding is no longer required.

To confirm that HilD directly binds the $m c p C$ promoter region, we used an electrophoretic mobility shift assay (EMSA). Purified maltose-binding protein HilD fusion protein (MBP-HilD) bound to the full-length $387 \mathrm{bp} m c p C$ promoter (P $m c p C-387$, Fig. 2e) but not to the shortened $79 \mathrm{bp}$ regulatory region (P $m c p C-79$, Fig. 2f) or the promoter region of another chemoreceptor (P $m c p B-333)$. Thus, binding is specific to the $m c p C$ promoter sequence and requires the region between -79 and -387 .

HilD can displace the global repressor H-NS from $\mathrm{DNA}^{32}$ and H-NS-binding sites are located near $m c p C^{33,34}$. We found that purified H-NS bound to the region upstream of $m c p C$ ( $\mathrm{P} m c p C$ 387) but not to the promoter of $m c p B$ (P $m c p B-333)$ (Fig. 3a, compare lanes 1 and 3). Furthermore, in a competitive EMSA HilD displaced H-NS from the $m c p C$ promoter (lanes 3-7) with full displacement occurring at 50-100 pM (lanes 5 and 6). Thus, HilD and H-NS specifically bind to the regulatory region of $m c p C$ and HilD is an antagonist of H-NS binding.

We next analyzed the effects of $\mathrm{H}-\mathrm{NS}$ in bacteria, using inducible expression of either WT (H-NSWT) or dominant negative $\mathrm{H}-\mathrm{NS}\left(\mathrm{H}-\mathrm{NS} \mathrm{Q}^{\mathrm{Q} 2 \mathrm{am}}\right)^{35}$ in the presence of $\mathrm{P} m c p \mathrm{C}-387$ $g f p$. However, since H-NS is required for expression of FliA, the sigma factor required for transcription of $m c p C^{36,37}$ (Fig. 2) we altered the reporter to replace the FliA ( $\sigma 28)$ recognition site with a $\sigma 70$ recognition sequence. This FliA-independent ( $\sigma 70$ dependent) $m c p C-g f p$ reporter $(\mathrm{P} m c p C-387(\sigma 70))$ displayed similar expression kinetics and magnitude to that of the original $m c p C$ reporter (Fig. $3 \mathrm{~b}$ ) and no longer required FliA or FlhD (Fig. 3c). Removing FlhD did have a slight detrimental effect on reporter expression, probably due to the loss of the flagellar protein FliZ and its positive impacts on HilD activity ${ }^{22}$. Importantly, $g f p$ expression was still dependent on HilD and amplified in the $\mathrm{HilD}^{+}$strain (Fig. 3c). Manipulation of H-NS 
Table 1 RNA sequencing results comparing WT and hilD $\Delta 3^{\prime} U T R$.

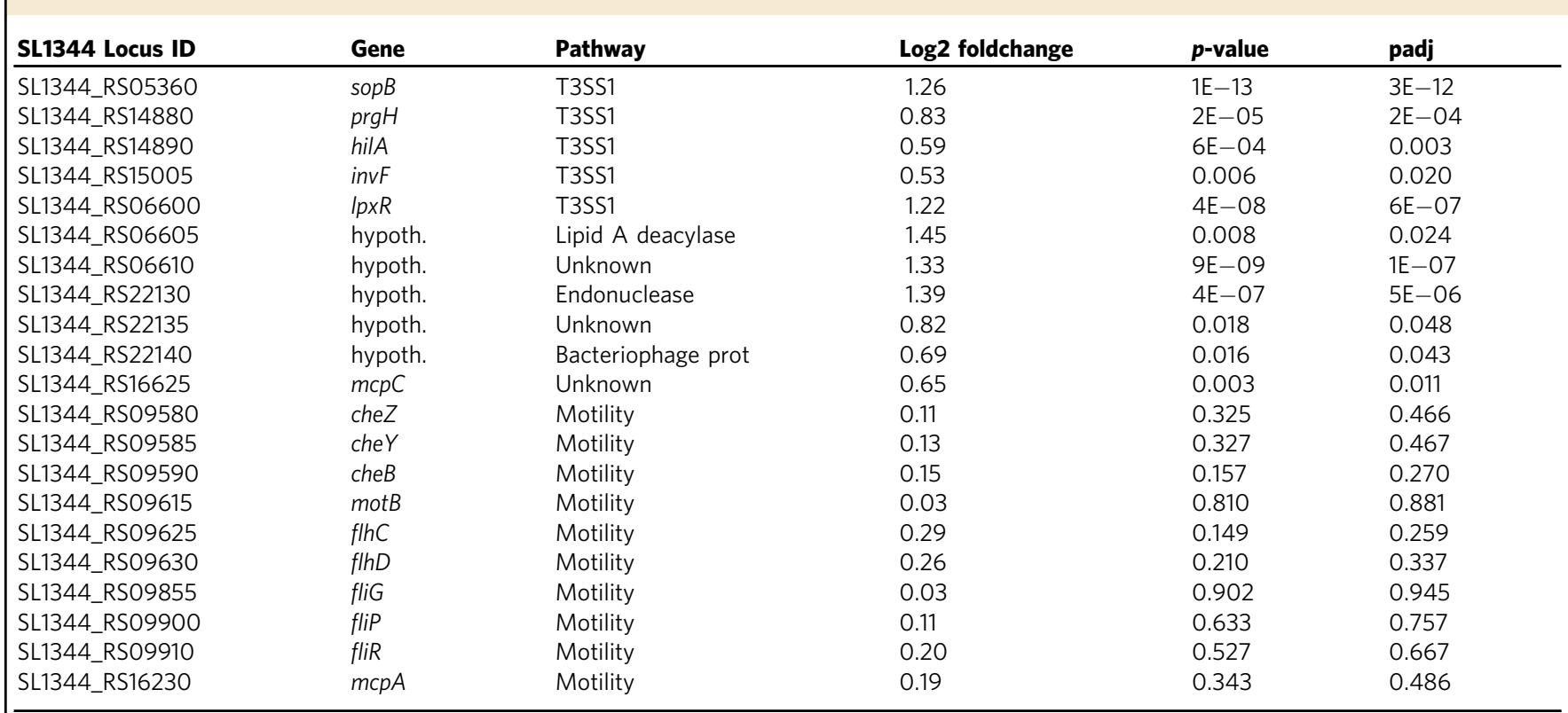

Shown are selected genes of the T3SS1, previously identified HilD targets and all upregulated motility genes. $n=3$ independent samples were collected from each strain. A positive log fold change indicates expression is up in hilD $\triangle 3^{\prime} U T R$ compared to WT. Significant gene expression differences were identified using DESeq2; $p$ values shown were calculated using the Wald test and the $p$ value was indicates expression is up in hilD $\Delta 3^{\prime} U T R$ compared to WT. Significant gene expression differences were identified using DESeq2; $p$ values shown
adjusted (padj) for multiple testing using the Benjamini and Hochberg procedure. Full RNAseq results are found in Supplementary Data 1.

a

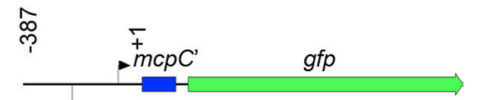

HilD ChIP
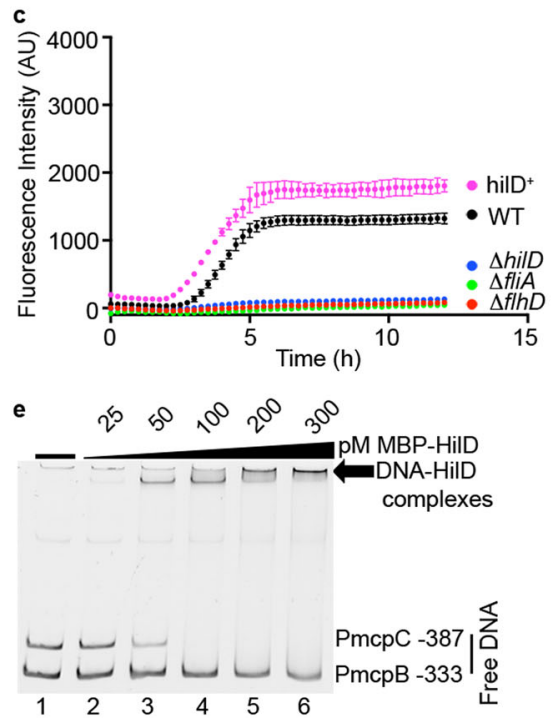

b
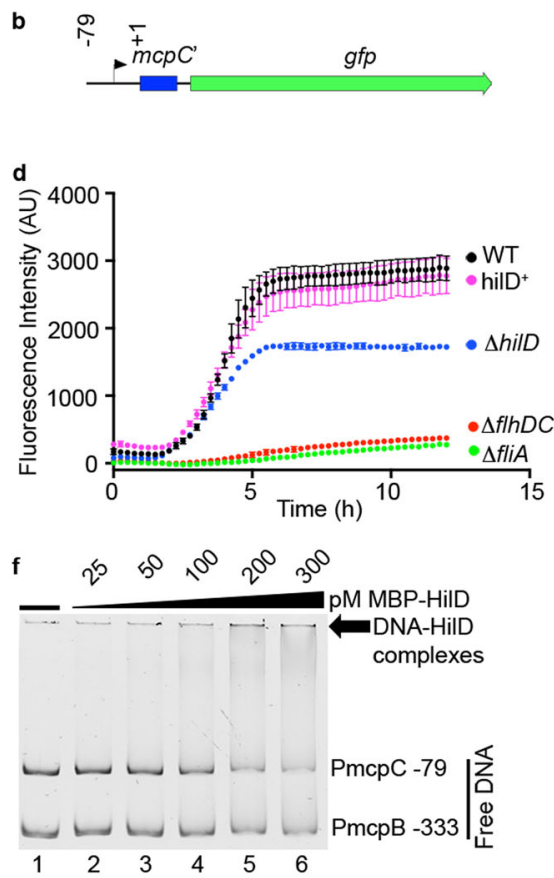

Fig. 2 mcpC is regulated by a Class III flagellar promoter and dependent on HilD. a, b Schematic of PmcpC-gfp transcriptional reporters: a PmcpC -387 gfp and $\mathbf{b}$ PmcpC $-79 \mathrm{gfp}$. Numbers indicate the nucleotide locations with reference to the start ATG $(+1) . m c p C^{\prime}$ represents the first 93 nucleotides of coding sequence of $m c p C$. The arrow indicates the predicted Class III promoter. HilD ChIP indicates the peak position of HilD binding identified by ref. 28 . c, $\mathbf{d}$ GFP fluorescence over time of the indicated strains harboring PmcpC -387 gfp (c) or PmcpC -79 gfp (d). Shown are the mean \pm SD of $n=3$ independent experiments for all strains except $\Delta f$ liA $(n=1)$. e, $\mathbf{f}$ Electrophoretic mobility shift assay (EMSA) of the indicated promoter regions with purified MBP-HilD. Images are representative of $n=3$ independent experiments. Source data are provided as a Source Data file.

levels by induction of $\mathrm{H}-\mathrm{NS} \mathrm{WT}^{\mathrm{T}}$ or $\mathrm{H}-\mathrm{NS} \mathrm{Q} 92 \mathrm{am}$ revealed that increasing $\mathrm{H}-\mathrm{NS}$ reduced $\mathrm{P} m c p C-387$ ( $\sigma 70)$ gfp expression, while decreasing $\mathrm{H}-\mathrm{NS}$ resulted in HilD-independent expression
(Fig. 3d). These results unequivocally demonstrate that H-NS represses $m c p C$ expression and HilD is required to derepress $m c p C$ expression under these conditions. 

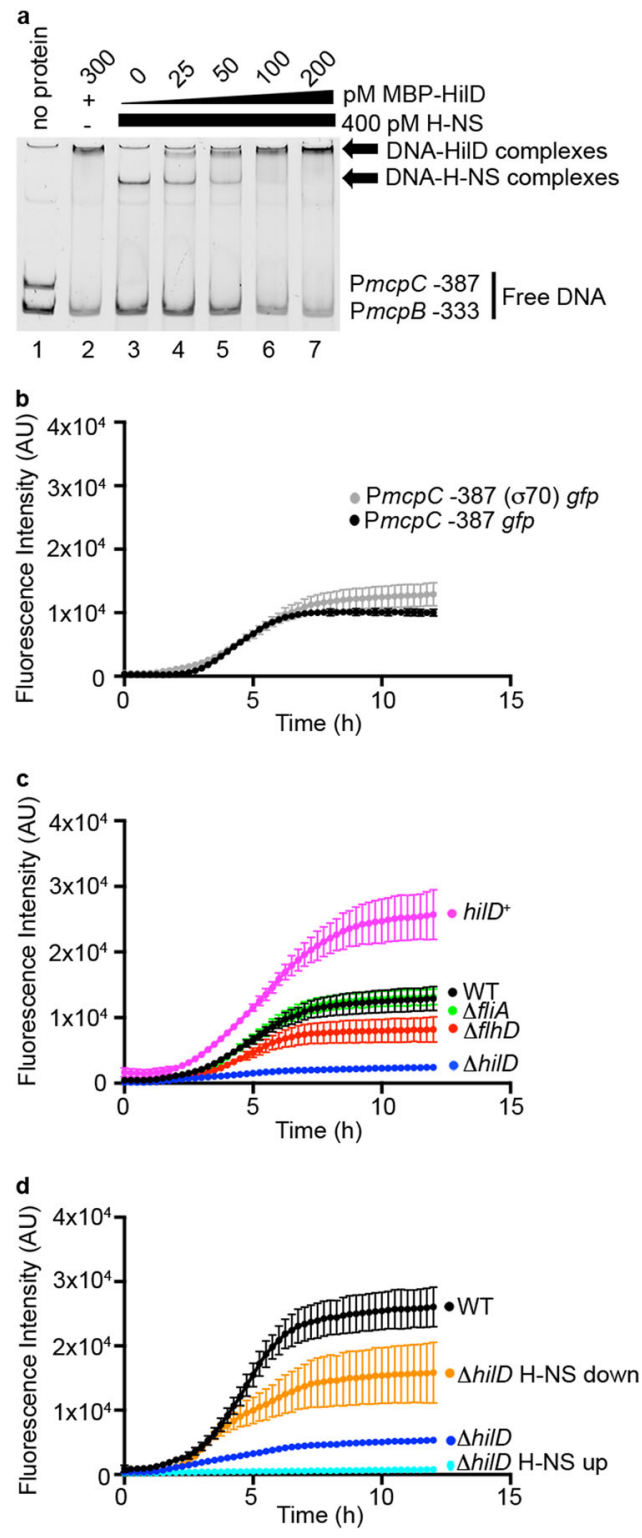

Fig. 3 HilD derepresses $\mathbf{m c p} \boldsymbol{C}$ by displacement of H-NS. a Competitive EMSA of the indicated promoter regions with purified MBP-HilD and H-NS. H-NS was added first (lanes 3-7) and increasing amounts of MBP-HilD was subsequently added (lanes 4-7). Similar results were obtained with $n=3$ independent experiments. b-d GFP fluorescence over time. b WT bacteria containing the indicated reporter. Shown are the mean \pm SD of $n=3$ independent experiments. c Indicated strains harboring PmcpC ( $\sigma 70)$ gfp. Shown are the mean \pm SD of $n=3$ independent experiments. $\mathbf{d}$ Indicated strains harboring PmcpC $(\sigma 70)$ gfp. H-NS up contains both PmcpC $(\sigma 70)$ gfp and pMPMT6 hnsWT. H-NS down contains both PmcpC ( $\sigma 70)$ gfp and pMPMT6 hns Q92am. Source data are provided as a Source Data file.

McpC functions independently of T3SS1 to increase bacteria/ host association. We hypothesized that $\mathrm{McpC}$ promotes chemotaxis of T3SS1-primed bacteria toward target host cells. To test this, we first ruled out that the invasion defect of $\triangle m c p C$ is due to aberrant expression or functionality of T3SS1. We evaluated SPI1 gene expression with a $p r g H-g f p[\mathrm{LVA}]$ reporter in WT vs. $\Delta m c p C$ by flow cytometry and found no differences in the percentage or magnitude of expression (Fig. 4a). Additionally, gentle centrifugation to increase contact of $\Delta m c p C$ with host cells overcame the invasion defect similar to rescuing a fliC mutant and unlike $\triangle$ SPI1 (Fig. 4c). Furthermore, the size of T3SS1-induced ruffles in
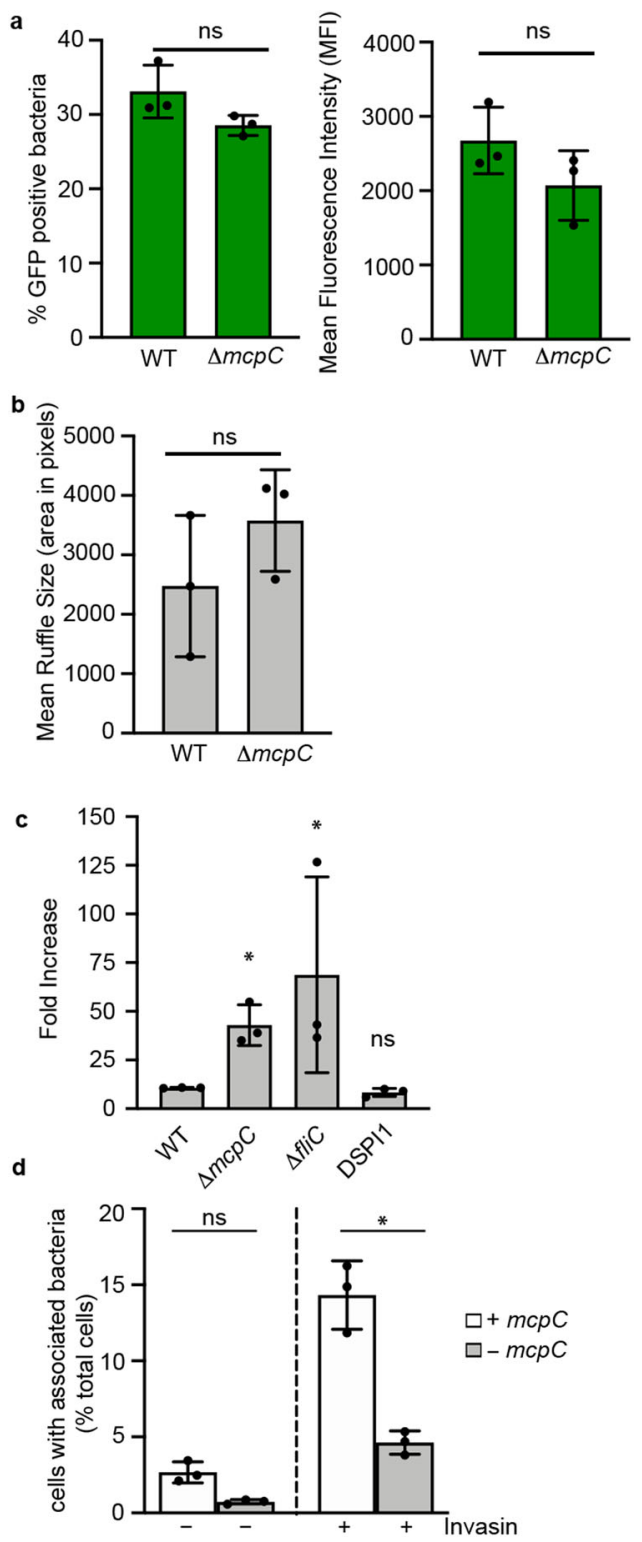

HeLa cells were similar when cells were infected by $\Delta m c p C$ vs. WT (Fig. 4b), indicating similar magnitude of effector delivery. Altogether, these results indicate that the invasion defect of $\triangle m c p C$ is due to decreased contact with host cells; once contact is made, normal T3SS1-dependent invasion occurs. To further confirm that $\mathrm{McpC}$ is important pre-invasion, we measured the ability of bacteria to make contact with host cells. Heterologous expression of the adhesin invasin from Yersinia pseudotuberculosis in the invasion deficient STm strain, $\triangle i n v A$, mediates tight attachment to host cells via interactions between invasin and $\beta 1$ chain integrin receptors ${ }^{38}$. As expected, invasin expression by STm increased the percentage of cells with associated bacteria over the $\triangle i n v A$ mutant alone, indicating that invasin was mediating attachment (Fig. 4d). However, when $m c p C$ was deleted from these strains, bacterial attachment was reduced. These results indicate McpC optimizes interaction with host cells.

$\mathrm{McpC}$ is required for optimal colonization in the gastrointestinal tract. To investigate if $\mathrm{McpC}$ confers an in vivo advantage, C57BL/6 mice were infected with a 1:1 mixture of wild type and $\triangle m c p C$. At 2 days post-infection (pi), the competitive 
Fig. 4 McpC functions independently of T3SS1 to increase bacterial/host cell association. a T3SS1 gene expression is not altered in $\triangle m c p C$. SPI1induced bacteria harboring PprgH gfp [LVA] were analyzed by flow cytometry ( $n=3$ independent experiments). The mean \pm SD percentage of GFP-positive bacteria (left panel) and the mean fluorescence intensity (right panel) are shown. Significance was determined using a two-tailed, paired Student's $t$ test (WT vs. $\Delta m c p C$, \% GFP positive: $p=0.2$, WT vs. $\Delta m c p C, M F I: p=0.1)$. b HeLa cells were infected with WT and $\Delta m c p C$ using centrifugation to synchronize bacterial/host association. Shown are the mean \pm SD ruffle sizes from WT infected cells ( $n=132$ cells, examined over three independent experiments) and from $\Delta m c p C$ infected cells $(n=157$ cells, examined over three independent experiments). Significance was determined using a two-tailed, paired Student's $t$ test (WT vs. $\Delta m c p C ; p=$ 0.4). c HeLa cells were infected with the indicated strains with or without centrifugation. Shown are the mean \pm SD fold increases in internalization with centrifugation at $1.5 \mathrm{~h}$ pi from $n=3$ independent experiments. Significance was determined by two-way Anova of log-transformed values followed by Tukey's multiple comparisons (WT vs. $\Delta m c p C ; p=0.002$, WT vs. $\Delta$ flic; $p=0.03$, WT vs. $\Delta$ SPI1: $p=0.7)$. d HeLa cells were infected with $\triangle i n v A$ or $\Delta i n v A / \triangle m c p C$, with or without expression of invasin (as indicated) for $6 \mathrm{~min}$. Shown are the mean \pm SD percentage of cells with associated bacteria ( $\Delta$ inv $A ; n=1212$ cells examined over three independent experiments, $\Delta i n v A+$ Invasin; $n=1518$ cells examined over three independent experiments, $\Delta i n v A / \Delta m c p C ; n=1173$ cells examined over three independent experiments, $\Delta i n v A / \Delta m c p C+$ Invasin; $n=1489$ cells examined over three independent experiments). Significance was determined by one-way Anova followed by Tukey's multiple comparisons $(\Delta$ inv $A+$ Invasin vs. $\Delta i n v A / \Delta m c p C+$ Invasin; $p=0.0001$. Green bars indicate that GFP-positive bacteria were analyzed in the assay. ns not significant, ${ }^{\star} p<0.05$. Source data are provided as a Source Data file.

index (CI) showed that $\Delta m c p C$ was outcompeted by the WT in the cecum, feces, and terminal ileum after oral inoculation of streptomycin pre-treated mice. However, after intravenous inoculation, both strains were recovered in equal amounts 4 days pi in the spleen. Furthermore, an $m c p C$ complemented strain was recovered in similar amounts to WT in the gut (Fig. 5a). This indicates that $\mathrm{McpC}$ plays an important role in the gastrointestinal tract but not during systemic infection of mice.

The calf ileal loop model ${ }^{39,40}$ has demonstrated a requirement for chemotaxis in the inflamed intestine ${ }^{1}$. Since $\mathrm{McpC}$ has a role in early "pre-inflammatory" colonization in the mouse gastrointestinal tract (Fig. 5a), we modified the established calf loop model by using a low inoculum $\left(10^{7} \mathrm{cfu} / \mathrm{loop}\right)$, focusing on an earlier time point $(2 \mathrm{~h} \mathrm{pi}$ ) and using a $\mathrm{CI}$ approach to maximize sensitivity. For identification by fluorescence microscopy, either WT or $\Delta m c p C$ expressed mCherry constitutively. In both jejunal and ileal loops, irrespective of which strain expressed mCherry, $\triangle m c p C$ was recovered in lower amounts than WT in gentamicintreated tissue but in equal amounts from luminal fluid and mucus (Fig. 5b). Confocal microscopy confirmed that more WT bacteria associated with host cells than the mutant although quantification was not possible due to low numbers of bacteria in the tissues (Supplementary Figs. 1 and 2). These data confirm that, in vivo, $m c p C$ is important for invasion of gut epithelium.

McpC functions to promote CCW flagellar rotational bias in SPI1-induced bacteria. If chemotactic signaling by McpC optimizes invasion, then disruption of all chemotaxis signaling should recapitulate a $\Delta m c p C$ invasion phenotype. To test this, we constructed a cheY mutant, which is unable to promote flagellar motor reversals (locked smooth), and a cheB mutant, which overproduces phosphorylated CheY (locked tumbly). $\Delta$ cheY had
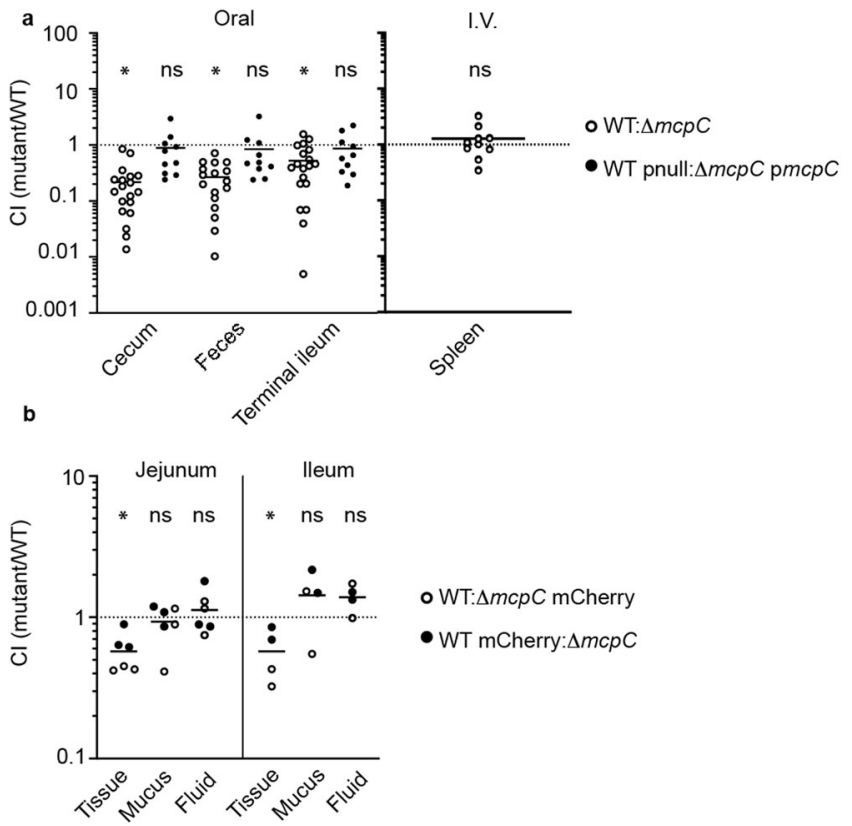

Fig. 5 McpC contributes to invasion in the gastrointestinal tract. a Streptomycin pretreated C57BL/6 mice were infected orally with a 1:1 mixture of $10^{6} \mathrm{CFU}$ (oral) or $500 \mathrm{CFU}$ (i.v.) each of either WT and $\Delta m c p C$ or WT carrying an empty plasmid (pnull) and $\triangle m c p C$ carrying a complementing plasmid $(\mathrm{pmcpC})$. For oral infections, the indicated organ CFU loads of either $n=10$ mice (WT pnull: $\Delta m c p C p m c p C$ ) or $n=20$ mice (WT: $\triangle m c p C$ ), were assessed at $2 \mathrm{~d}$ pi. For intravenous infection, the spleen CFU loads of $n=10$ mice (WT: $\Delta m c p C$ ) were assessed at $4 \mathrm{~d}$ pi. Each dot represents the $\mathrm{Cl}$ from a single mouse with the mean shown. Statistical significances were determined by two-way Anova followed by Sudak's multiple comparisons on log-transformed CFU values for analyzing multiple tissue types in the gastrointestinal tract (WT vs. $\Delta m c p C$ in the cecum: $p=$ 0.0001 , in the feces: $p=0.0002$, in the terminal ileum: $p=0.005$; WT pnull: $\triangle m c p C$ pmcpC in the cecum: $p=0.5$, in the feces: $p=0.3$, in the terminal ileum: $p=0.5$ ). For analyzing statistical significance in the spleen, a two-tailed Wilcoxon test was performed on log-transformed CFU values (WT vs. $\Delta m c p C: p=0.13$ ). b Ligated jejunal ( $n=6$ loops from 3 calves) or ileal loops ( $n=4$ loops from 2 calves) were injected with a 1:1 mixture of $10^{7} \mathrm{CFU}$ total of WT and $\triangle m c p C$, one of which expressed mCherry as indicated. Each dot represents the $\mathrm{Cl}$ from an individual loop with the mean shown. Tissue samples (gentamicin-treated biopsies), mucus, or luminal fluid were assessed at $2 \mathrm{~h}$ pi. Statistical significance was determined by two-way Anova followed by Sudak's multiple comparisons on logtransformed CFU values (WT vs. $\triangle m c p C$ in the jejunal tissue: $p=0.007$, in the ileal tissue: $p=0.02$, in the jejunal fluid: 0.9 , in the ileal fluid: $p=0.9$, in the jejunal mucus: $p=0.8$, in the ileal mucus: $p=0.9$ ). ns $=$ not significant, ${ }^{\star} p<0.05$. Source data are provided as a Source Data file.

no invasion defect in HeLa cells, indicating chemotactic signaling is not required for invasion (Fig. 6a). Conversely, overproduction of phosphorylated $\mathrm{CheY}$ in $\Delta c h e B$ resulted in a large invasion defect. Intriguingly, $\Delta m c p C / \Delta c h e Y$ had no defect, indicating that forced smooth swimming can rescue $\triangle m c p C$ invasion. These results suggest that $\mathrm{McpC}$ prevents motor reversals and promotes smooth swimming.

Since $\triangle m c p C$ has only a slight motility defect on agar swim plates $^{41}$, possibly because of McpC dependence on SPI1 induction (Fig. 2), we used light microscopy to assess flagella rotational bias in SPI1-induced free-swimming bacteria. We used PprgH-gfp [LVA] to selectively track and analyze swimming patterns of SPI1-induced $\mathrm{GFP}^{+}$bacteria $^{42}$. Approx. $31 \%$ of WT tracks contained more than two angle changes $\left(>30^{\circ}\right)$, while $\sim 57 \%$ of 


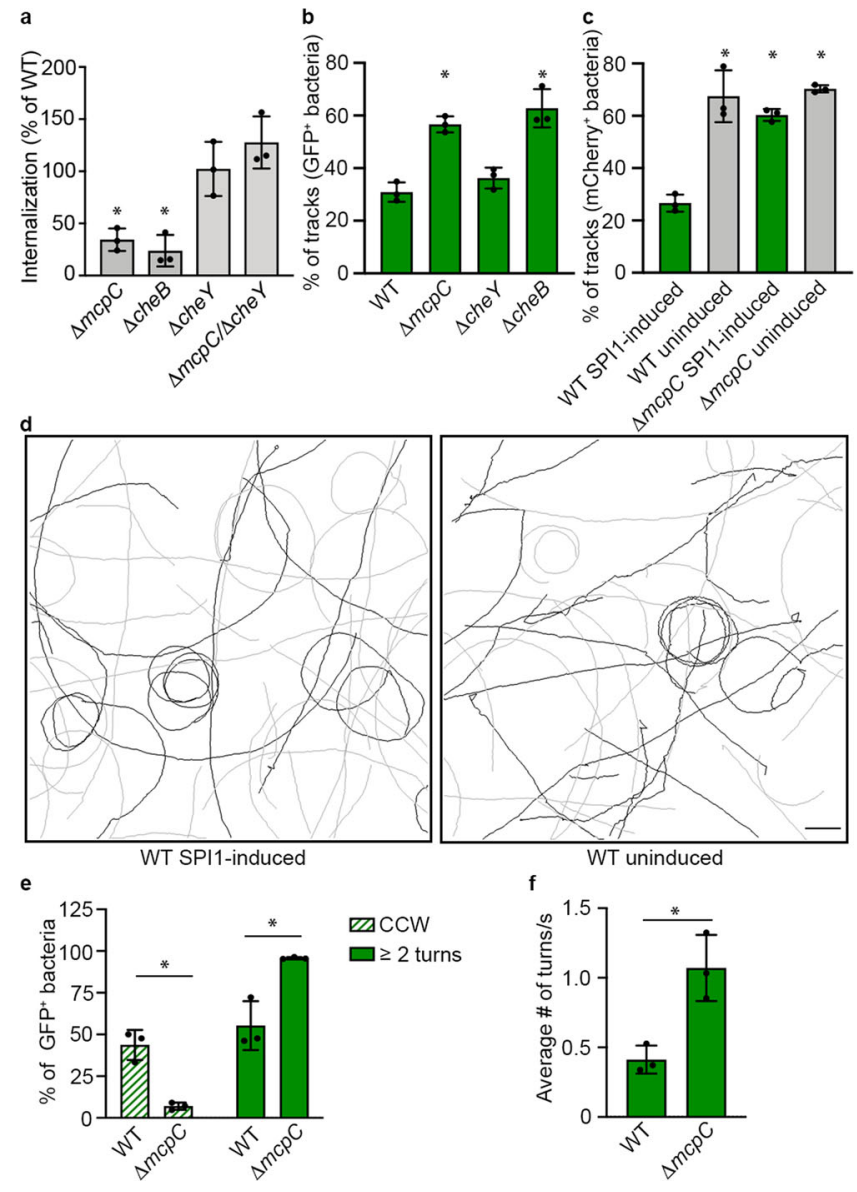

$\triangle m c p C$ tracks did (Fig. 6b). Furthermore, swimming behavior of $\mathrm{GFP}^{+}$WT bacteria was indistinguishable from the $\Delta c h e Y$ mutant (smooth), while $\triangle m c p C$ bacteria were identical to a cheB mutant (tumbly) (Fig. 6b). Thus SPI1-induced WT STm have a "locked smooth" swimming phenotype that is dependent on expression of $m c p C$. If this is true, then non SPI1-induced bacteria should not be "locked smooth". In order to simultaneously track free-swimming $\mathrm{GFP}^{-}$and $\mathrm{GFP}^{+}$bacteria, we added constitutive mCherry expression to construct dual P prgH-gfp [LVA]. Comparison of mCherry ${ }^{+} / \mathrm{GFP}^{+}$bacteria (SPI1-induced) vs. mCherry ${ }^{+} / \mathrm{GFP}^{-}$bacteria (non-SPI1-induced) revealed that non-SPI1-induced WT bacteria (Fig. 6c, d) behave similarly to the $m c p C$ and $c h e B$ mutants (Fig. 6b). In contrast, SPI1-induced and non-SPI1-induced $\triangle m c p C$ behaviors were indistinguishable, further confirming the requirement for $\mathrm{McpC}$ in this phenotype (Fig. 6c). Surprisingly, these swimming phenotypes were observed in the absence of added ligands, and a high-throughput screen failed to identify any ligands (Supplementary Data 2). Smooth swimming occurs when all motors spin CCW resulting in bundled flagella. Cells tumble when one or more motors switch to $\mathrm{CW}$, resulting in dispersal of the attached filament from the bundle. E. coli cells can tumble when one motor switches to CW; however, Salmonella has more flagella than E. coli $i^{43,44}$ and it is unclear whether a single motor reversal in Salmonella will cause a tumble or less obvious disruptions to smooth swimming (Fig. 6d). To analyze single motors, we performed flagellar tether assays ${ }^{45}$ using PprgH-gfp[LVA] to identify SPI1-induced bacteria. More $\mathrm{GFP}^{+}$WT bacteria had motors displaying exclusively CCW rotation compared to $\mathrm{GFP}^{+} \Delta m c p C$, which reversed more often (Fig. 6e). Additionally, the frequency of turns in reversing motors was higher in $\mathrm{GFP}^{+} \Delta m c p C$ than $\mathrm{GFP}^{+}$WT (Fig. 6f). Thus, the
Fig. $\mathbf{6}$ McpC promotes CCW flagellar rotational bias in SPI1-induced

bacteria. a HeLa cells were infected with the indicated strains for $1.5 \mathrm{~h} \mathrm{pi}$. Shown is the mean \pm SD inoculum normalized to WT of $n=3$ independent experiments. Statistical significance was determined by one-way Anova followed by Tukey's multiple comparisons (WT vs. $\Delta m c p C: p=0.009$, WT vs. $\Delta$ cheB: $p=0.003$, WT vs. $\Delta$ cheY: $p=0.9$, WT vs. $\Delta m c p C / \Delta$ cheY: $p=$ $0.4)$. b, c Swimming behavior of the indicated strains harboring either (b) PprgH-gfp[LVA] or (c) dual PprgH-gfp[LVA]. b Shown is the mean \pm SD percentage of GFP+ (SPI1-induced) bacteria with tracks containing $>2$ angle changes for $n=3$ individual experiments in which 2872 WT bacteria, $2038 \Delta m c p C$ bacteria, $947 \Delta c h e B$ bacteria, and $2654 \Delta c h e Y$ bacteria were examined. Statistical significance was determined by one-way Anova followed by Tukey's multiple comparisons (WT vs. $\Delta m c p C$ : $p=0.0008$, WT vs. $\Delta$ cheY: $p=0.6$, WT vs. $\Delta$ cheB: $p=0.0002$ ). c Shown is the mean \pm SD percentage of tracks containing $>2$ angle changes for $n=3$ individual experiments in which 1625 WT SPI1-induced bacteria, 789 WT uninduced bacteria, 907 SPI1-induced $\triangle m c p C$ bacteria, and $470 \triangle m c p C$ uninduced bacteria were examined. Statistical significance was determined by oneway Anova followed by Tukey's multiple comparisons (WT SPI1-induced vs. WT uninduced: $p=0.0001$, WT SPI1-induced vs. $\triangle m c p C$ SPI1-induced: $p=0.0003$, WT SPI1-induced vs. $\Delta m c p C$ uninduced: $p=0.0001$ ). d Representative tracks from SPI1-induced mCherry+GFP+ WT bacteria (left) and tracks from uninduced mCherry ${ }^{+}$GFP- $^{-}$WT bacteria (right). Gray tracks contain $\leq$ two angle changes, black tracks contain $>2$ angle changes. Scale bar, $20 \mu \mathrm{m}$. e, f Flagella tethering assay. SPI1-induced bacteria harboring PprgH-gfp[LVA] were tethered to glass and imaged. Shown are the mean \pm SD $\%$ of GFP-positive bacteria that had exclusively CCW rotation (hatched bars) or GFP-positive bacteria that reversed two or more times during the $15 \mathrm{~s}$ observation period (solid bars). Data are from $n=3$ independent experiments in which 65 WT bacteria and $71 \Delta m c p C$ bacteria were examined. Statistical significance was determined using two-way Anova followed by Sidak's multiple comparisons (WT vs $\Delta m c p C$ exclusively CCW: $p=0.002$, WT vs $\Delta m c p C$ with $2+$ turns: $p=0.009$ ). (f) Shown is the mean \pm SD number of turns/s for the dataset in e. Statistical significance was determined by a two-tailed paired Student's $t$-test (WT vs. $\triangle m c p C: p=0.02$ ). Green indicates that GFP-positive bacteria were analyzed in the assay. ns $=$ not significant, ${ }^{\star} P<0.05$. Source data are provided as a Source Data file.

SPI1 transcriptional program includes a HilD-dependent mechanism to promote unstimulated extended smooth swimming via McpC.

\section{Discussion}

Here we identified a chemoreceptor, McpC, as a critical component in early colonization of the mammalian gut. By promoting smooth swimming in SPI1-induced "invasive" bacteria, McpC maximizes interaction with, and invasion of, the intestinal epithelium in the uninflamed gut. Although flagellar motility exists in both T3SS1-expressing and non-expressing cells, we found that swimming behavior is different for SPI1-induced bacteria (those that go on to colonize host epithelium) vs. non-SPI1-induced bacteria (presumably those that replicate and thrive within the gut lumen). Under SPI1-inducing conditions, HilD derepresses $m c p C$ by displacement of $\mathrm{H}-\mathrm{NS}$ from its promoter region. Production of McpC results in CCW bias and a smooth swimming phenotype that facilitates invasion. In the GI tract, SPI1-induced STm are predominantly located close to the mucosal surface ${ }^{11,46}$. We propose that McpC maximizes the chance of these invasive bacteria interacting with their target cells via a process that involves near surface swimming 47,48 . On the other hand, nonSPI1-induced STm, which do not express McpC, retain the 
normal chemotactic functions that are required to thrive in the lumen of the inflamed gut ${ }^{1,2,49-51}$.

Smooth swimming is induced when chemoreceptors bind an attractant, and is typically followed by rapid adaptation, mediated by the methyl-transferase/-esterase pair CheBR, ensuring that the bacteria are rapidly reset ${ }^{52}$. However, we were unable to identify a ligand for $\mathrm{McpC}$ and indeed, in our experimental assays for swimming behavior and flagellar rotation, no exogenous ligands were required. This suggests that a potential McpC ligand could be an intrinsic molecule or a form of energy sensing. Alternatively, the intrinsic conformational structure of McpC may mimic a ligand bound state. To our knowledge, such a bacterial chemoreceptor has not been identified in nature, however replacement of single residues at key locations in the model chemotaxis receptor Tsr mimic ligand binding, likely through changes in the protein structure ${ }^{53}$. If $\mathrm{McpC}$ has acquired such a mutation, repression by H-NS would effectively limit any detrimental effects on chemotaxis in non-SPI1-induced bacteria. In addition, the long-lived signature of $\mathrm{McpC}$-induced smooth swimming indicates a lack of, or poor, adaptation. Indeed, McpC does not contain the conserved $\mathrm{NWE}^{\mathrm{T}} / \mathrm{s}$ F pentapeptide motif that recruits CheBR, and thus may require "adaptation assistance" by other chemoreceptors ${ }^{54,55}$. Thus, we propose McpC induces smooth swimming via a non-canonical mechanism that is ligandindependent and resistant to adaptation.

It was hypothesized nearly 30 years ago that chemotaxis and invasion would share common regulatory elements in Salmonella ${ }^{3}$. Smooth swimming mutants have increased invasion in cultured epithelial cells whereas tumbly mutants, which reverse direction frequently, have reduced invasion ${ }^{3-5}$. Our work has identified McpC as the link between invasiveness and smooth swimming in STm, the expression of which is coordinated with the T3SS1 and flagella by the master regulator HilD. Interestingly, naturally occurring mutations in chemotaxis genes, which potentially further increase smooth swimming, have been identified in the human restricted Salmonella Paratyphi A and Typhi as well as in the highly invasive zoonotic serovar Cholerasuis ${ }^{56,57}$. Additionally, smooth swimming mutants of some Vibrio species also display hypervirulence ${ }^{58,59}$ and it has been hypothesized that repression of chemotaxis gene expression, leading to smooth swimming, may be responsible for the hypervirulence of Vibrio cholerae shed in rice water stools ${ }^{60,61}$. The fact that genetically unrelated enteric pathogens coordinate smooth swimming with invasiveness indicates this may be a more widespread virulence strategy. While the regulatory and functional mechanisms need further study, this is a potential target for the development of novel antimicrobial therapies.

\footnotetext{
Methods

Bacterial cultures and growth conditions. STm SL1344 was used in all experiments (Supplementary Table 1). Strain stocks were frozen in $15 \%$ glycerol and stored at $-80^{\circ} \mathrm{C}$. Strains were streaked onto LB agar supplemented with appropriate antibiotics and stored at $4{ }^{\circ} \mathrm{C}$ for up to 1 week.
}

SPI1-induction. As previously described ${ }^{12}$, SPI1 induction was as follows: Overnight $(16-18 \mathrm{~h})$ cultures were prepared by inoculating one colony into $2 \mathrm{~mL} \mathrm{LB}$ Miller broth (US Biological, Animal Free) (10 g/L salt) with selective antibiotics, in a loose-capped $14 \mathrm{~mL}$ polypropylene round-bottom tube (Becton Dickinson) and incubated at $37^{\circ} \mathrm{C}$ with shaking $(225 \mathrm{rpm})$. To induce SPI1, $0.3 \mathrm{~mL}$ of the overnight culture was inoculated into $10 \mathrm{~mL}$ LB-Miller broth (no antibiotics), in a $125 \mathrm{~mL}$ Erlenmeyer flask for $3.5 \mathrm{~h}$ at $37^{\circ} \mathrm{C}$ with shaking $(225 \mathrm{rpm})$.

Animal inoculations. For mouse experiments, bacteria were grown in a $125 \mathrm{~mL}$ Erlenmeyer flask in $10 \mathrm{~mL}$ LB-Miller broth containing the appropriate antibiotic for $18 \mathrm{~h}$ at $37^{\circ} \mathrm{C}$ with shaking $(225 \mathrm{rpm})$ and diluted in sterile pharmaceutical grade saline (SPGS). For calf experiments, loose-capped $14 \mathrm{~mL}$ polypropylene round-bottom tubes (Becton Dickinson) were used for both overnight cultures and subcultures. One colony was inoculated into $5 \mathrm{~mL}$ LB-Miller broth with selective antibiotics and incubated for $14 \mathrm{~h}$ at $37^{\circ} \mathrm{C}$ with shaking $(225 \mathrm{rpm})$, then diluted
1:100 in $5 \mathrm{~mL} \mathrm{LB}$-Miller without antibiotics and sub-cultured for $4 \mathrm{~h}$ at $37^{\circ} \mathrm{C}$ with shaking $(225 \mathrm{rpm})$. Cultures were diluted in LB-Miller for inoculation.

Growth curves and transcriptional reporter assays. Overnight cultures of STm were diluted 1:25 and $200 \mu \mathrm{L}$ was aliquoted in triplicate into 96-well plates and grown in an Infinite 200 Pro plate-reader (Tecan). Plates were shaken at $37^{\circ} \mathrm{C}$. OD600 and fluorescence (Ex 478, Em 515) were read every $15 \mathrm{~min}$. OD600 and fluorescence values from blank media were subtracted from the average of triplicate wells for each sample.

Mammalian cells. HeLa (human cervical adenocarcinoma, ATCC) cells were grown at $37^{\circ} \mathrm{C}$ in $5 \% \mathrm{CO}_{2}$ in complete growth medium: Eagle's minimal essential medium (Mediatech) supplemented with $10 \%(\mathrm{v} / \mathrm{v})$ heat-inactivated fetal bovine serum (FBS) (Thermo Fisher), $2 \mathrm{mM}$ L-glutamine and $1 \mathrm{mM}$ sodium pyruvate. Caco2 subclone C2BBe1 (human colorectal adenocarcinoma, ATCC CRL-2012) were grown in Dulbecco's minimal essential medium (DMEM) supplemented with $10 \%(\mathrm{v} / \mathrm{v})$ heat-inactivated FBS, $4 \mathrm{mM} \mathrm{L}$-glutamine and $10 \mu \mathrm{g} / \mathrm{mL}$ human transferrin. Cells were passaged as recommended by ATCC and used for experiments within 15 passages of receipt. Human peripheral blood monocytes, enriched by apheresis, were obtained from peripheral blood provided by the Department for Transfusion Medicine and the National Institutes of Health Clinical Center at the National Institutes of Health (Bethesda, MD). Monocytes were further enriched by centrifugation with Ficoll-Paque (GE Healthcare) and then resuspended in a freezing medium of $10 \%$ dimethyl sulfoxide (DMSO)-90\% FBS at $10^{8}$ cells $/ \mathrm{mL}$ and stored in liquid $\mathrm{N}_{2}$. Monocytes were purified with the Dynabeads Untouched human monocytes kit (Thermo Fisher); the purity of recovered cells was routinely $>90 \%$ CD14+ by flow cytometry. These cells were plated in complete medium containing RPMI 1640 medium (Gibco), $1 \mathrm{mM}$ sodium pyruvate, $1 \times$ MEM nonessential amino acids, $10 \mathrm{mM}$ HEPES buffer, $2 \mathrm{mM}$ glutamate, $5 \%$ (vol/vol) heatinactivated human male AB serum (HuS) (Sigma-Aldrich), and $100 \mathrm{ng} / \mathrm{mL}$ human recombinant macrophage colony-stimulating factor (M-CSF) (PeproTech). Cells were grown in $5 \% \mathrm{CO}_{2}$ at $37^{\circ} \mathrm{C}$ and used for assays on day 7 . On days 3 and $5,50 \%$ of the volume of the cultures was replaced with fresh complete RPMI, 5\% serum, and $200 \mathrm{ng} / \mathrm{mL}$ M-CSF. All data are from three independent experiments using cells prepared on different days from different donors.

Construction of chromosomal deletion mutants. The bacteriophage $\lambda$ Red recombinase system was used for construction of gene disruption mutants in $\mathrm{STm}^{62}$. Mutants were verified by PCR analysis, and the mutation transduced by P22 back to WT SL1344. If necessary, antibiotic cassettes were removed using pCP20 before combining mutations using P22 transduction.

Plasmid construction. All plasmid ligation reactions were carried out using T4 DNA ligase (Promega). Restriction enzymes were obtained from New England Biolabs (NEB). The high-fidelity polymerase Phusion was used for all PCR reactions (NEB). PCR primers were sourced from Integrated DNA technologies. All plasmid constructs were verified by sequencing. Plasmids are listed in Supplementary Table 1. Oligonucleotide sequences are listed in Supplementary Table 2.

Plasmid backbones. A synthetic transcriptional terminator (Part Bba_B0015 of the Registry of Standard Biological Parts (parts.igem.org)) was cloned into the pWSK29 2 Plac KpnI site using primers B0015 KpnI F and B0015 KpnI R. Likewise, the terminator was added to pMPMA3 $\triangle$ Plac using the NotI and SacII sites with the primers B0015 NotI F and B0015 SacII R.

Transcriptional reporters. Promoter regions (positions with respect to start ATG), $m c p C$ ( -387 to +93$)$ and $(-79$ to +93$)$ were PCR amplified with PmcpC $-387 \mathrm{XbaI} F$ or $\mathrm{P} m c p C-79 \mathrm{XbaI} F$ and $m c p C$ stop KpnI R (which introduces a stop codon) and ligated to a $\mathrm{XbaI} / K p n \mathrm{I}$ digest of pGFP[LVA] (Clontech). This produces an operon-like fusion, in which the transcribed mRNA codes for a short $m c p C$ peptide prior to a stop codon, followed by a strong ribosome-binding site (RBS) and $g f p[\mathrm{LVA}]$. The fusion was then amplified without the LVA tag using primers P $m c p C-387 \mathrm{XbaI} F$ or P $m c p C-79 \mathrm{XbaI} F$ and $g f p$ no LVA HindIII R and

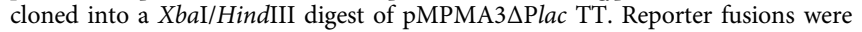
moved into a plasmid with a compatible origin (ori pMB1) to that of the hns expressing plasmids (pMPMT6 ori p15A) to enable maintenance of both plasmids in STm. To do this, the $m c p C-387$ to +93 gfp fusion was amplified with PmcpC -387 EcoRV F and B0015 SacI R and ligated into an EcoRV/SacI digest of pBAD18-Cm, which replaces the $\mathrm{P}_{\mathrm{BAD}}$ promoter and most of the araC gene, resulting in $\mathrm{pMB} 1 \mathrm{P} m c p C-387 g f p$. In order to change the sigma factor recognition site, a gene fragment containing the change (Supplementary Table 2) was synthesized (Integrated DNA Technologies) to replace the fragment between EcoRV and PmlI sites of pMB1 PmcpC - 387 gfp. To construct dual PprgH-gfp [LVA], the ProC-mCherry-TT expression cassette was PCR amplified from pCON1-ProC.mCherry and cloned into the NotI and SacI sites of pMPMA3 3 Plac PprgH-gfp[LVA]. 
Expression constructs. $m c p C$ gene was amplified using $\mathrm{P} m c p C-387 \mathrm{Xba \textrm {I } F}$ and $m c p C$ ORF NotI R and cloned into XbaI/NotI digested WSK29 $\Delta$ Plac TT, resulting in pmcpC. The inv locus was excised from pRI20363 using BamHI and cloned into pWSK29 in the same direction as the Plac promoter, resulting in pWSK29-inv. The hilD gene including its $3^{\prime}$-UTR was PCR amplified from SL1344 genomic DNA using primers hilD NheI RBS F and hilD 3'-UTR SphI R and cloned into the NheI/ $S p h I$ sites of a low copy arabinose-inducible expression plasmid (pMPMA3 $\triangle \mathrm{Plac}$ $\mathrm{p}_{\mathrm{BAD}}$ ) made by subcloning the $\mathrm{araC}$ gene and promoter from $\mathrm{pBAD} 18-\mathrm{Cm}$ into pMPMA3 $\triangle$ Plac using ClaI and HindIII.

Gentamicin protection assay. These assays were done similar to previous studies $^{64,65}$. Briefly, human monocytes were seeded in 96 -well plates at $4 \times 10^{4}$ cells per well, differentiated as described above, and used for infection on day 7. Immortalized cell lines were seeded $20-24 \mathrm{~h}$ prior to infection in 24 -well plates at $4.5 \times 10^{4}$ cells (HeLa) or $5.5 \times 10^{4}$ per well (C2Bbe1). C2Bbel were seeded onto collagen-coated plates. Late-log phase cultures (SPI1-induced) STm were collected by centrifugation at $8000 \times g$ for $2 \mathrm{~min}$, washed and resuspended in Hank's buffered saline solution (HBSS) and used immediately to infect cells for $10 \mathrm{~min}$ at an MOI of $\sim 50$ for epithelial cells and $\sim 10$ for human macrophages. T3SS-1 mutants were used at an MOI of $\sim 30$ in human macrophages. Centrifugation at $500 \times g$ for 5 min was not used except in Fig. 4. Extracellular bacteria were removed by washing with HBSS and cells were incubated in antibiotic-free complete growth media until 30 min pi. Cells were then incubated for either $15 \mathrm{~min}$ (macrophages) or $1 \mathrm{~h}$ (epithelial cells) in complete growth media supplemented with L-histidine $(500 \mu \mathrm{g} / \mathrm{mL})$ and gentamicin $(50 \mu \mathrm{g} / \mathrm{mL})$. The media was then replaced by complete growth media supplemented with L-histidine $(500 \mu \mathrm{g} / \mathrm{mL})$ and gentamicin $(10 \mu \mathrm{g} / \mathrm{mL})$ for the remainder of the infection. At indicated time-points, monolayers were lysed in $0.2 \%(\mathrm{w} / \mathrm{v})$ sodium deoxycholate in PBS and viable intracellular bacteria were enumerated by plating on LB agar. Internalization was calculated as the $\%$ of the inoculum remaining after the gentamicin protection assay and normalized to WT.

Protein purification. MBP-HilD and MBP-H-NS were expressed in E. coli BL21/ DE3 containing pMAL-hilD or pMAL-hns and purified by using an amylose column per manufacturer's instructions (NEB). After purification, the buffer was

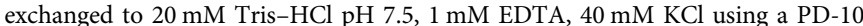
desalting column (Amersham). Protein concentration was determined using a BCA assay (Bio-Rad) and proteins were stored in aliquots in this buffer with $50 \%$ glycerol and $1 \mathrm{mM}$ DTT at $-20^{\circ} \mathrm{C}$. The MBP was cleaved off of MBP-H-NS using Factor Xa protease per manufacturer's instructions (NEB). Recombinant ligandbinding domain of McpC (amino acid residues 35-189) or Tar (amino acid residues $34-190)$ including $\mathrm{N}$-terminal $6 \mathrm{x}$ His tags on each were commercially purified (GenScript).

Electrophoretic mobility shift assays (EMSAs). DNA fragments were PCR amplified from SL1344 genomic DNA using the following primer pairs: $\mathrm{P} m c p C$ $-387-+93: \mathrm{P} m c p C-387 \mathrm{XbaI} F$ and PmcpC stop KpnI R; P $m c p C-79-+93$ : $\mathrm{P} m c p C-79$ XbaI F and P $m c p C$ stop KpnI R; P $m c p B-333-+45$ :P $m c p B$ XbaI F and $\mathrm{P} m c p B$ stop KpnI R. PCR products were purified using the QIAquick PCR purification kit (Qiagen). Reactions contained $100 \mathrm{ng}$ of PCR product with increasing concentrations of purified MBP-HilD or H-NS in binding buffer containing $10 \mathrm{mM}$ Tris- $\mathrm{HCl}(\mathrm{pH} 8), 50 \mathrm{mM} \mathrm{KCl}, 1 \mathrm{mM}$ dithiothreitol (DTT), $0.5 \mathrm{mM}$ EDTA, 5\% glycerol, and $10 \mu \mathrm{g} / \mathrm{mL}$ bovine serum albumin (BSA), in a total volume of $20 \mu \mathrm{L}$. Reactions were incubated for $20 \mathrm{~min}$ at RT and then separated by electrophoresis in $6 \%$ nondenaturing polyacrylamide gels in $0.5 \times$ Tris-borate-EDTA (TBE) buffer. In order to differentiate the size of MBP-HilD-DNA complexes from H-NS-DNA complexes in competitive EMSA gels, MBP was first cleaved from MBP-H-NS using Factor Xa protease (NEB). For competitive EMSAs, the DNA fragment was first incubated with $0.4 \mu \mathrm{M}$ H-NS for $15 \mathrm{~min}$ and then incubated with increasing concentrations of MBP-HilD for an additional $20 \mathrm{~min}$. Gels were stained with SYBR safe (Thermo Fisher) and visualized with an alpha-imager UV transilluminator (Bio-Rad).

Ligand screening. The dimerization status of purified ligand-binding domain of $\mathrm{McpC}$ was assessed commercially using sedimentation velocity analytical ultracentrifugation (SV-AUC) (KBI BioPharma) and revealed that dimers were present at $\geq 4 \mathrm{mg} / \mathrm{mL}$. Thermal shift assays were done as previously described ${ }^{66}$ using a BioRad CFX Real-Time PCR instrument in a 384-well format. Ligands were prepared by dissolving Biolog PM compounds in $50 \mu \mathrm{L}$ water to obtain a final concentration of 10-20 mM, except for PM4A which was dissolved in $20 \mu \mathrm{L}$. A $10 \mathrm{mM}$ solution of L-cystine was also prepared in $50 \mathrm{mM} \mathrm{HCl}$. Each $10 \mu \mathrm{L}$ reaction contained $4 \mathrm{mg} / \mathrm{mL}$ protein $(\sim 212 \mu \mathrm{M}), 10 \times$ SYPRO Orange dye (Thermo Fisher) in a $50 \mathrm{mM}$ HEPES buffer $\mathrm{pH} 8.2 \mu \mathrm{L}$ of ligand was added to each well. As a positive control, Tar LBD was used at $10 \mu \mathrm{M}$ protein with $1 \mathrm{mM}$ L-aspartate. Samples were heated by 1 degree per min from $25-75^{\circ} \mathrm{C}$. Protein unfolding curves were monitored by fluorescence of SYPRO Orange. Melting temperatures were calculated using non-linear fitting to the Boltzmann equation (GraphPad).

RNA sequencing. Total RNA was quantified using Quant-it RiboGreen assay (Thermo Fisher) and $500 \mathrm{ng}$ total RNA was depleted of ribosomal RNAs using
RiboZero bacterial rRNA depletion kit, following the manufacturer's recommended procedure (Epicentre). Ribosomal-depleted RNAs were purified using RNAClean XP beads (Beckman Coulter Life Sciences) and eluted in Fragment, Prime, and Finish buffer found in Illumina TruSeq Stranded mRNA Library Preparation Kit (Illumina Inc). This kit was then used to generate sequencing libraries beginning at the fragmentation step with no other modifications. Each sample was given a unique molecular barcode and fragment-sized using a BioAnalyzer High Sensitivity chip (Agilent). The samples were quantitated using Kapa Library Quant kit (Illumina) Universal qPCR mix (Kapa Biosystems) and diluted to a 2 nanomolar working concentration. Equal volumes were combined into a single pool and clustered across a RAPID 2-lane flowcell. The flowcell was then sequenced on an Illumina HiSeq 2500 instrument for 60 cycles in one direction with an additional seven cycles to sequence the molecular barcodes, generating an average of 13.5 million reads per sample.

Raw fastq reads were trimmed of Illumina adapter sequencing using a proprietary script and then trimmed and filtered for quality using the FASTXToolkit (Hannon Lab, CSHL). Remaining reads were mapped to the Salmonella enterica subsp. enterica serovar Typhimurium str. SL1344 genome (chromosome and plasmids); NC_016810.1, NC_017718.1, NC_017719.1, and NC_017720.1 using Bowtie ${ }^{67}$ with parameters --score-min $\mathrm{L}, 0,-0.15$. Reads mapping to genes were counted using htseq-count ${ }^{68}$. The Bioconductor package DESeq2 ${ }^{69}$ was used for data normalization and differential gene expression analysis. Data were from three independent samples from each strain.

\section{Immunofluorescence microscopy}

Cultured cells. HeLa cells were plated on glass coverslips in 24-well plates $\left(5.5 \times 10^{4}\right.$ cells per well). For cell association assays using pWSK29-inv, cells were infected as described above except for $6 \mathrm{~min}$ instead of $10 \mathrm{~min}$. Infected cells were washed three times with ice-cold HBSS and fixed in $2.5 \%(\mathrm{w} / \mathrm{v})$ paraformaldehyde (PFA) for $10 \mathrm{~min}$ at $37^{\circ} \mathrm{C}$, followed by three washes in PBS. To differentially stain intracellular and extracellular bacteria, monolayers were blocked (but not permeabilized) in $10 \%$ normal donkey serum in PBS, then incubated with goat antiSalmonella CSA-1 (KPL) which had been conjugated to Pacific Blue (Thermo Fisher) per manufacturer's instructions (1:250) to bind extracellular bacteria. Cells were washed three times, then permeabilized with $0.1 \%(\mathrm{w} / \mathrm{v})$ saponin plus $10 \%$ $(\mathrm{v} / \mathrm{v})$ normal goat serum in PBS. Cells were then incubated with goat anti-Salmonella CSA-1 which had been conjugated to AlexaFluor 488 (Thermo Fisher) per manufacturer's instructions (1:250) which will now bind both extracellular and intracellular bacteria, along with AlexaFluor 568-conjugated Phalloidin (ThermoFisher) (1:40) to stain cellular F-actin. Coverslips were then washed sequentially with PBS and distilled water then mounted on glass slides in a Mowiol 4-88 solution supplemented with $2.5 \%$ (w/v) DABCO (Sigma-Aldrich) ${ }^{65}$. The total number of cells with associated bacteria was counted. For evaluation of ruffle size, GFP-expressing bacteria were used to infect cells as described above except with an MOI of $\sim 10$ and contact with host cells was synchronized by centrifugation at 500 $\times g$ for $5 \mathrm{~min}$ at $37^{\circ} \mathrm{C}$. Immediately after centrifugation, cells were washed thrice with warm HBSS and fixed in $2.5 \%$ PFA as described above. Cells were incubated in $0.1 \%$ saponin in PBS followed by staining with AlexaFluor 568-conjugated Phalloidin and mounting as described above. Images were captured with the same gain and exposure for each sample on a DS-Qi2 camera using a $\times 60$ objective on a Nikon Ti2 epifluorescence widefield microscope. Post-acquisition analysis of ruffle size was done using ImageJ software. Ruffle size was determined as follows: Images were converted to 8-bit binary $(0,255)$ images and a threshold was placed on the phalloidin channel. A circular ROI measuring $150 \times 150$ pixels was centered on an individual bacterium associated with phalloidin. Ruffles with more than one associated bacterial cell were not measured. Integrated density in the phalloidin channel within the ROI was measured and divided by 255 to calculate the intensity/ pixel area.

Calf tissue. Flash frozen $6 \mathrm{~mm}$ biopsy punches were thawed/fixed in ice-cold Carnoy's buffer $(60 \% \mathrm{MeOH}, 30 \%$ acetic acid, $10 \%$ acetic acid) for $3 \mathrm{~h}$ on ice before washing as follows: $2 \times \mathrm{MeOH}$ washes, $30 \mathrm{~min}$ each, $2 \times \mathrm{ETOH}$ washes, 20 min each, $2 \times$ PBS washes, $20 \mathrm{~min}$ each. Tissues were then embedded and frozen in OCT media and $5 \mu \mathrm{m}$ sections were cut and placed on slides for staining. Tissue sections were blocked with $2 \%$ donkey serum, $1 \%$ BSA, $0.1 \%$ Triton X-100, $0.05 \%$ Tween-20 in PBS, following by staining with the following primary antibodies: mouse anticytokeratin clone C-51 (Thermo Fisher, 1:100), rabbit anti-mCherry polyclonal (Thermo Fisher, 1:100), and goat anti-Salmonella CSA-1 (1:100) conjugated to AlexaFluor 488 (Thermo Fisher) per manufacturer's instructions. Secondary antibodies (1:500) were AlexaFluor 568-conjugated donkey anti-rabbit (Thermo Fisher) and $\mathrm{CF}^{\circ} 633$ conjugated donkey anti-mouse (Biotium). After staining, slides were stained for 10 min with wheat germ agglutinin (WGA) conjugated to $\mathrm{CF}^{\circ} 405$ (Biotium, 1:500), followed by fixation with $2.5 \%$ PFA for 10 min. Samples were mounted with ProLong Gold with DAPI (Thermo Fisher). Images were captured using a Zeiss LSM 710 confocal laser-scanning microscope with either a Plan APOCHROMAT $\times 63 / 1.4$ N.A. objective or a $\times 20 / 0.8$ N.A. objective.

Tethered bacteria. Flagellar rotation patterns were determined by a cell tethering assay ${ }^{70}$. SPI1-induced bacteria were passed through a 27 -gauge needle $\sim 50 \times$ to shear flagella, followed by two washes in motility buffer $(10 \mathrm{mM}$ potassium 
phosphate $\mathrm{pH} 7,0.1 \mathrm{mM}$ EDTA). Bacteria were then diluted to $\sim 1.5 \times 10^{8} / \mathrm{mL}$ in tethering buffer $(10 \mathrm{mM}$ potassium phosphate $\mathrm{pH} 7,0.1 \mathrm{mM}$ EDTA, $10 \mathrm{mM}$ sodium lactate, $75 \mathrm{mM}$ sodium chloride, and $0.1 \mathrm{mM}$ L-methionine). $0.1 \mathrm{~mL}$ of bacterial suspension was mixed with $2 \mu \mathrm{l}$ of anti-Hi antiserum (SSI Diagnostica) and placed in black glass bottom 24-well plates for $10 \mathrm{~min}$ at RT. Unbound bacteria were removed, and fresh tethering buffer was placed in the wells. $15 \mathrm{~s}$ movies were captured with either a Nikon Ti or Ti2 epifluorescence widefield microscope using a Plan Apo VC ×60/1.4 N.A. objective and an Orca Flash 4.0 camera (Hamamatsu) at 200 frames per second (fps). Movies were sub-stacked to $100 \mathrm{fps}$ and exported for spot detection and tracking analysis in Imaris 9.5.0 software. XY position information for each track was exported and data was processed using in-house python script. Spin direction of tethered bacteria from frame $j$ to frame $j+1$ was determined by calculating the cross-product of the two positional vectors $\vec{R}$ with the given $x$ and $y$ coordinates of each vector as in Eq. (1):

$$
\vec{R}_{j} \times \vec{R}_{j+1}=\left(R_{j}^{x *} R_{j+1}^{y}\right)-\left(R_{j}^{y *} R_{j+1}^{x}\right)
$$

The sign of the resultant cross product indicates direction the bacteria is spinning. A direction change was scored if the direction of spin changed from one frame to the next. Direction changes per second were calculated as the total number of direction changes divided by the total number of frames in movie for each bacterium, multiplied by frames per second of movie.

Swimming pattern analysis. SPI1-induced cultures were diluted 1:500 into tethering buffer, mixed gently and $100 \mu \mathrm{L} /$ well was placed in black 96 -well glass bottom plates. $15 \mathrm{~s}$ movies were captured with a Nikon Ti2 epifluorescence widefield microscope using a Plan Fluor Ph2 DLL $\times 40 / 0.75$ N.A. objective and an Orca Flash 4.0 camera (Hamamatsu) at $30 \mathrm{fps}$. For simultaneous capture of GFP and mCherry, a DC2 beam splitter (Photometrics) was used with two iXon Ultra cameras (Andor). Individual bacteria were tracked using Imaris 9.5.0. $X Y$ position data was exported and used to calculate the angle of direction change from frame $j$ to $j+1$. The velocity vector $\vec{V}$ for each frame $j$ containing two components is Eq. (2):

$$
\vec{V}_{j}=\left[V_{j}^{x}, V_{j}^{y}\right]
$$

where each instantaneous velocity components for $\vec{V}_{j}$ are calculated from the positional vectors $\vec{R}$ of the neighboring frames as in Eqs. (3) and (4):

$$
\begin{gathered}
V_{j}^{x}=\frac{\mathrm{d} R_{j}^{x}}{\mathrm{~d} t}=\frac{2}{3 T}\left(R_{j+1}^{x}-R_{j-1}^{x}\right)-\frac{1}{12 T}\left(R_{j+2}^{x}-R_{j-2}^{x}\right) \\
V_{j}^{y}=\frac{\mathrm{d} R_{j}^{y}}{\mathrm{~d} t}=\frac{2}{3 T}\left(R_{j+1}^{y}-R_{j-1}^{y}\right)-\frac{1}{12 T}\left(R_{j+2}^{y}-R_{j-2}^{y}\right)
\end{gathered}
$$

The angle in degrees by which the bacterium changes direction between frames $j$ and $j+1$ is then calculated in Eq. (5):

$$
\theta^{\circ}=\cos ^{-1}\left(\frac{\left(V_{j}^{x} * V_{j+1}^{x}\right)+\left(V_{j}^{y} * V_{j+1}^{y}\right)}{\left\|V_{j}\right\| *\left\|V_{j+1}\right\|}\right) * \frac{180}{\pi}
$$

where $\left\|V_{j}\right\|$ and $\left\|V_{j+1}\right\|$ are the magnitudes of velocity vector $\vec{V}_{j}$ and $\vec{V}_{j+1}$. Strains were compared by calculating the percentage of tracks with two or more angle changes of at least $30^{\circ}$ each. Each experiment compiled results from five movies per strain.

Flow cytometry. $10-20 \mu \mathrm{L}$ bacteria were fixed in $500 \mu \mathrm{L} 2.5 \%$ (w/v) paraformaldehyde at RT for $10 \mathrm{~min}$, centrifuged and finally washed once in PBS. Bacteria were then stained with $10 \mu \mathrm{M}$ Syto41 (Thermo Fisher) in PBS for $30 \mathrm{~min}$ at RT, washed once with PBS by centrifugation, and resuspended in $1 \mathrm{~mL}$ PBS for analysis on a BD LSR II flow cytometer (BD Bioscience). Data were analyzed using FlowJo software (Tree Star). Samples were gated on Syto $41^{+}$events and the \% and mean intensity of $\mathrm{GFP}^{+}$events was measured. Gating strategy is shown in Supplementary Information.

Mouse experiments. The C57BL/6 mice used in this study were either from a colony of mice originally purchased from The Jackson Laboratory (Bar Harbor, ME) and maintained at the Rocky Mountain Laboratories or purchased from The Jackson Laboratory and used shortly after arrival. Animal facilities maintained the following parameters: temperature, $72 \pm 3^{\circ} \mathrm{F}$; humidity, $50 \pm 10 \%$; dark/light cycle, 12:12 h.

For oral infection, mice were streptomycin treated $24 \mathrm{~h}$ before infection, using a blunt end gavage needle with $100 \mu \mathrm{l}$ SPGS containing $200 \mathrm{mg} / \mathrm{mL}$ streptomycin. Mice were fasted for $4 \mathrm{~h}$ prior to all gavages. Mice were gavaged or infected intraveneously by retroorbital injection with a volume of $100 \mu \mathrm{l}$. Mice were euthanized by isoflurane inhalation followed by exsanguination. Tissues were collected in screwcap tubes containing $500 \mu \mathrm{l}$ SPGS and 3-4 $2.0 \mathrm{~mm}$ zirconia beads (BioSpec Products) and homogenized using a Bead Mill 24 (Fisher Scientific, 4.85 $\mathrm{m} / \mathrm{s}$ for $20 \mathrm{~s}$ ). Tubes were weighed before and after organ collection. CFUs were estimated by $10 \mu \mathrm{l}$ spot plating of 10 -fold dilutions on LB agar plates containing the appropriate antibiotic.

Bovine ligated jejunal and ileal loop surgeries. The ligated jejunal and ileal loop proceedures were similar to previous studies with some important changes ${ }^{11}$. Calves 4-6 weeks of age were obtained from the Texas A\&M University Veterinary Medical Park and received colostrum prior to isolation. Animals were fed antibiotic-free milk replacer twice daily and water ad libitum. Prior to surgery, calves were tested for Salmonella spp. in fecal excretions. Calves were fasted $12 \mathrm{~h}$ before surgery. After laparotomy, the distal jejunum and ileum were externalized and loops, $\sim 6 \mathrm{~cm}$ in length, were formed with $1-\mathrm{cm}$ spacer loops in-between. Bacterial cultures of $1 \mathrm{~mL}$ LB-Miller containing $10^{7}$ total CFU were prepared as described above and loaded into a $3 \mathrm{~mL}$ syringe with a 26 -gauge needle and kept on ice until inoculation into the loop via intraluminal injection. Following inoculation, the loops were returned to the body cavity and maintained at $\sim 37^{\circ} \mathrm{C}$. At $2 \mathrm{~h} \mathrm{pi}$, loops were excised and processed. Briefly, loops were weighed and opened, and luminal fluid collected. Mucus was collected by gently stamping a $10 \mathrm{~mm}$ circular filter paper onto the tissue surface before immersion of the filter into SPGS. Six mm tissue biopsy punches were either flash frozen or placed into SPGS containing 100 $\mu \mathrm{g} / \mathrm{mL}$ gentamicin for $1 \mathrm{~h}$ before washing twice and placing into fresh SPGS. All samples were collected or ultimately placed into screwcap tubes containing SPGS and 3-4 $2.0 \mathrm{~mm}$ zirconia beads (BioSpec Products) and homogenized using a Bead Mill 24 (Fisher Scientific, $4.85 \mathrm{~m} / \mathrm{s}$ for $20 \mathrm{~s}$ ). Tubes were weighed before and after sample collection and CFUs were estimated by plating on LB agar plates containing the appropriate antibiotic. Loops from three independent calves were utilizedy.

Reporting summary. Further information on research design is available in the Nature Research Reporting Summary linked to this article.

\section{Data availability}

The RNA sequencing data has been deposited in NCBI's Gene Expression Omnibus and are accessible at https://www.ncbi.nlm.nih.gov/geo/query/acc.cgi?acc=GSE156765. All other data supporting the findings of this study are available within the paper and the Supplementary Information. Source data are provided with this paper.

Received: 15 September 2020; Accepted: 27 November 2020; Published online: 13 January 2021

\section{References}

1. Rivera-Chávez, F. et al. Salmonella uses energy taxis to benefit from intestinal inflammation. PLoS Pathog. 9, e1003267 (2013).

2. Stecher, B. et al. Motility allows S. Typhimurium to benefit from the mucosal defence. Cell. Microbiol. 10, 1166-1180 (2008).

3. Jones, B. D., Lee, C. A. \& Falkow, S. Invasion by Salmonella typhimurium is affected by the direction of flagellar rotation. Infect. Immun. 60, 2475-2480 (1992).

4. Khoramian-Falsafi, T., Harayama, S., Kutsukake, K. \& Pechère, J. C. Effect of motility and chemotaxis on the invasion of Salmonella typhimurium into HeLa cells. Microb. Pathog. 9, 47-53 (1990).

5. Hoffmann, S., Schmidt, C., Walter, S., Bender, J. K. \& Gerlach, R. G. Scarless deletion of up to seven methyl-accepting chemotaxis genes with an optimized method highlights key function of CheM in Salmonella Typhimurium. PLoS ONE 12, e0172630 (2017)

6. Lostroh, C. P. \& Lee, C. A. The Salmonella pathogenicity island-1 type III secretion system. Microbes Infect. 3, 1281-1291 (2001).

7. Stecher, B. et al. Flagella and chemotaxis are required for efficient induction of Salmonella enterica serovar Typhimurium colitis in streptomycin-pretreated mice. Infect. Immun. 72, 4138-4150 (2004).

8. Lou, L., Zhang, P., Piao, R. \& Wang, Y. Salmonella Pathogenicity Island 1 (SPI-1) and its complex regulatory network. Front. Cell. Infect. Microbiol. 9, 270 (2019).

9. Eade, C. R. et al. Salmonella Pathogenicity Island 1 is expressed in the chicken intestine and promotes bacterial proliferation. Infect. Immun. 87, 4879 (2019).

10. Boyen, F. et al. Salmonella Typhimurium SPI-1 genes promote intestinal but not tonsillar colonization in pigs. Microbes Infect. 8, 2899-2907 (2006).

11. Laughlin, R. C. et al. Spatial segregation of virulence gene expression during acute enteric infection with Salmonella enterica serovar Typhimurium. mBio 5, e00946-13 (2014).

12. Ibarra, J. A. et al. Induction of Salmonella pathogenicity island 1 under different growth conditions can affect Salmonella-host cell interactions in vitro. Microbiology 156, 1120-1133 (2010).

13. Sridhar, S. \& Steele-Mortimer, O. Inherent variability of growth media impacts the ability of Salmonella Typhimurium to interact with host cells. PLOS ONE 11, e0157043 (2016). 
14. Hamed, S. et al. Synergistic action of SPI-1 gene expression in Salmonella enterica serovar Typhimurium through transcriptional crosstalk with the flagellar system. BMC Microbiol. 19, 211-212 (2019).

15. Baxter, M. A. \& Jones, B. D. The fimYZ genes regulate Salmonella enterica Serovar Typhimurium invasion in addition to type 1 fimbrial expression and bacterial motility. Infect. Immun. 73, 1377-1385 (2005).

16. Ellermeier, J. R. \& Slauch, J. M. Adaptation to the host environment: regulation of the SPI1 type III secretion system in Salmonella enterica serovar Typhimurium. Curr. Opin. Microbiol. 10, 24-29 (2007).

17. Iyoda, S., Kamidoi, T., Hirose, K., Kutsukake, K. \& Watanabe, H. A flagellar gene fliZ regulates the expression of invasion genes and virulence phenotype in Salmonella enterica serovar Typhimurium. Microb. Pathog. 30, 81-90 (2001).

18. Lin, D., Rao, C. V. \& Slauch, J. M. The Salmonella SPI1 type three secretion system responds to periplasmic disulfide bond status via the flagellar apparatus and the RcsCDB system. J. Bacteriol. 190, 87-97 (2008).

19. Thijs, I. M. V. et al. Delineation of the Salmonella enterica serovar Typhimurium HilA regulon through genome-wide location and transcript analysis. J. Bacteriol. 189, 4587-4596 (2007).

20. Saini, S., Slauch, J. M., Aldridge, P. D. \& Rao, C. V. Role of cross talk in regulating the dynamic expression of the flagellar Salmonella pathogenicity island 1 and type 1 fimbrial genes. J. Bacteriol. 192, 5767-5777 (2010).

21. Singer, H. M., Kühne, C., Deditius, J. A., Hughes, K. T. \& Erhardt, M. The Salmonella Spil virulence regulatory protein HilD directly activates transcription of the flagellar master operon flhDC. J. Bacteriol. 196, 1448-1457 (2014).

22. Chubiz, J. E. C., Golubeva, Y. A., Lin, D., Miller, L. D. \& Slauch, J. M. FliZ regulates expression of the Salmonella pathogenicity island 1 invasion locus by controlling HilD protein activity in Salmonella enterica serovar typhimurium. J. Bacteriol. 192, 6261-6270 (2010).

23. Mouslim, C. \& Hughes, K. T. The effect of cell growth phase on the regulatory cross-talk between flagellar and Spil virulence gene expression. PLoS Pathog. 10, e1003987 (2014).

24. Ellermeier, C. D. \& Slauch, J. M. RtsA and RtsB coordinately regulate expression of the invasion and flagellar genes in Salmonella enterica serovar Typhimurium. J. Bacteriol. 185, 5096-5108 (2003).

25. Schechter, L. M. \& Lee, C. A. AraC/XylS family members, HilC and HilD, directly bind and derepress the Salmonella typhimurium hilA promoter. Mol. Microbiol. 40, 1289-1299 (2001)

26. Hung, C.-C., Haines, L. \& Altier, C. The flagellar regulator fliT represses Salmonella pathogenicity island 1 through flhDC and fliZ. PLoS ONE 7, e34220 (2012).

27. López-Garrido, J., Puerta-Fernández, E. \& Casadesús, J. A eukaryotic-like 3' untranslated region in Salmonella enterica hilD mRNA. Nucleic Acids Res. 42, 5894-5906 (2014).

28. Petrone, B. L., Stringer, A. M. \& Wade, J. T. Identification of HilD-regulated genes in Salmonella enterica serovar Typhimurium. J. Bacteriol. 196, 1094-1101 (2014).

29. Smith, C., Stringer, A. M., Mao, C., Palumbo, M. J. \& Wade, J. T. Mapping the regulatory network for Salmonella enterica Serovar Typhimurium Invasion. mBio 7, 263 (2016)

30. Frye, J. et al. Identification of new flagellar genes of Salmonella enterica serovar Typhimurium. J. Bacteriol. 188, 2233-2243 (2006).

31. Chilcott, G. S. \& Hughes, K. T. Coupling of flagellar gene expression to flagellar assembly in Salmonella enterica serovar Typhimurium and Escherichia coli. Microbiol. Mol. Biol. Rev. 64, 694-708 (2000).

32. Martínez, L. C., Banda, M. M., Fernández-Mora, M., Santana, F. J. \& Bustamante, V. H. HilD induces expression of Salmonella pathogenicity island 2 genes by displacing the global negative regulator $\mathrm{H}-\mathrm{NS}$ from ssrAB. $J$. Bacteriol. 196, 3746-3755 (2014).

33. Lucchini, S. et al. H-NS mediates the silencing of laterally acquired genes in bacteria. PLoS Pathog. 2, e81 (2006).

34. Navarre, W. W. et al. Selective silencing of foreign DNA with low GC content by the H-NS protein in Salmonella. Science 313, 236-238 (2006).

35. Bustamante, V. H. et al. HilD-mediated transcriptional cross-talk between SPI-1 and SPI-2. Proc. Natl Acad. Sci. USA 105, 14591-14596 (2008).

36. Bertin, P. et al. The H-NS protein is involved in the biogenesis of flagella in Escherichia coli. J. Bacteriol. 176, 5537-5540 (1994).

37. Ali, S. S. et al. Silencing by H-NS potentiated the evolution of Salmonella. PLoS Pathog. 10, e1004500 (2014).

38. Leong, J. M., Fournier, R. S. \& Isberg, R. R. Identification of the integrin binding domain of the Yersinia pseudotuberculosis invasin protein. EMBO J. 9, 1979-1989 (1990).

39. Higginson, E. E., Simon, R. \& Tennant, S. M. Animal models for Salmonellosis: applications in vaccine research. Clin. Vaccin. Immunol. 23, 746-756 (2016).

40. Tsolis, R. M., Xavier, M. N., Santos, R. L. \& Bäumler, A. J. How to become a top model: impact of animal experimentation on human Salmonella disease research. Infect. Immun. 79, 1806-1814 (2011).
41. Wang, Q., Mariconda, S., Suzuki, A., McClelland, M. \& Harshey, R. M. Uncovering a large set of genes that affect surface motility in Salmonella enterica Serovar Typhimurium. J. Bacteriol. 188, 7981-7984 (2006).

42. Berg, H. C. \& Brown, D. A. Chemotaxis in Escherichia coli analyzed by threedimensional tracking. Antibiot. Chemother. (1971) 19, 55-78 (1974).

43. Iino, T. Assembly of Salmonella flagellin in vitro and in vivo. J. Supramol. Struct. 2, 372-384 (1974).

44. Turner, L., Ryu, W. S. \& Berg, H. C. Real-time imaging of fluorescent flagellar filaments. J. Bacteriol. 182, 2793-2801 (2000).

45. Silverman, M. \& Simon, M. Flagellar rotation and the mechanism of bacterial motility. Nature 249, 73-74 (1974).

46. Hausmann, A. et al. Intestinal epithelial NAIP/NLRC4 restricts systemic dissemination of the adapted pathogen Salmonella Typhimurium due to sitespecific bacterial PAMP expression. Mucosal Immunol. 1-15 (2020). https:// doi.org/10.1038/s41385-019-0247-0

47. Misselwitz, B. et al. Near surface swimming of Salmonella Typhimurium explains target-site selection and cooperative invasion. PLoS Pathog. 8 , e1002810 (2012).

48. Furter, M., Sellin, M. E., Hansson, G. C. \& Hardt, W.-D. Mucus architecture and near-surface swimming affect distinct Salmonella Typhimurium infection patterns along the murine intestinal tract. Cell Rep. 27, 2665-2678.e3 (2019)

49. Schmitt, C. K. et al. Absence of all components of the flagellar export and synthesis machinery differentially alters virulence of Salmonella enterica serovar Typhimurium in models of typhoid fever, survival in macrophages, tissue culture invasiveness, and calf enterocolitis. Infect. Immun. 69, 5619-5625 (2001)

50. Winter, S. E. et al. Gut inflammation provides a respiratory electron acceptor for Salmonella. Nature 467, 426-429 (2010).

51. Rivera-Chávez, F. et al. Energy taxis toward host-derived nitrate supports a Salmonella Pathogenicity Island 1-independent mechanism of invasion. mBio 7, 1806 (2016).

52. Barnakov, A. N., Barnakova, L. A. \& Hazelbauer, G. L. Efficient adaptational demethylation of chemoreceptors requires the same enzymedocking site as efficient methylation. Proc. Natl Acad. Sci. USA 96, 10667-10672 (1999)

53. Kitanovic, S., Ames, P. \& Parkinson, J. S. Mutational analysis of the control cable that mediates transmembrane signaling in the Escherichia coli serine chemoreceptor. J. Bacteriol. 193, 5062-5072 (2011).

54. Feng, X., Baumgartner, J. W. \& Hazelbauer, G. L. High- and low-abundance chemoreceptors in Escherichia coli: differential activities associated with closely related cytoplasmic domains. J. Bacteriol. 179, 6714-6720 (1997).

55. Feng, X., Lilly, A. A. \& Hazelbauer, G. L. Enhanced function conferred on lowabundance chemoreceptor Trg by a methyltransferase-docking site. J. Bacteriol. 181, 3164-3171 (1999).

56. McClelland, M. et al. Comparison of genome degradation in Paratyphi A and Typhi, human-restricted serovars of Salmonella enterica that cause typhoid. Nat. Genet. 36, 1268-1274 (2004).

57. Chiu, C.-H. et al. The genome sequence of Salmonella enterica serovar Choleraesuis, a highly invasive and resistant zoonotic pathogen. Nucleic Acids Res. 33, 1690-1698 (2005)

58. Ushijima, B. \& Häse, C. C. Influence of chemotaxis and swimming patterns on the virulence of the coral pathogen Vibrio coralliilyticus. J. Bacteriol. 200, 1505 (2018).

59. Larsen, M. H. \& Boesen, H. T. Role of flagellum and chemotactic motility of Vibrio anguillarum for phagocytosis by and intracellular survival in fish macrophages. FEMS Microbiol. Lett. 203, 149-152 (2001)

60. Butler, S. M. \& Camilli, A. Both chemotaxis and net motility greatly influence the infectivity of Vibrio cholerae. Proc. Natl Acad. Sci. USA 101, 5018-5023 (2004).

61. Butler, S. M. et al. Cholera stool bacteria repress chemotaxis to increase infectivity. Mol. Microbiol. 60, 417-426 (2006).

62. Datsenko, K. A. \& Wanner, B. L. One-step inactivation of chromosomal genes in Escherichia coli K-12 using PCR products. Proc. Natl Acad. Sci. USA 97, 6640-6645 (2000)

63. Isberg, R. R., Voorhis, D. L. \& Falkow, S. Identification of invasin: a protein that allows enteric bacteria to penetrate cultured mammalian cells. Cell 50, 769-778 (1987).

64. Steele-Mortimer, O. Infection of epithelial cells with Salmonella enterica. Methods Mol. Biol. 431, 201-211 (2008).

65. Lathrop, S. K. et al. Salmonella Typhimurium infection of human monocytederived macrophages. Curr. Protoc. Microbiol 50, e56 (2018).

66. Ehrhardt, M. K. G., Warring, S. L. \& Gerth, M. L. Screening chemoreceptor-ligand interactions by high-throughput thermal-shift assays. Methods Mol. Biol. 1729, 281-290 (2018)

67. Langmead, B. \& Salzberg, S. L. Fast gapped-read alignment with Bowtie 2. Nat Methods 9, 357-359 (2012).

68. Anders, S., Pyl, P. T. \& Huber, W. HTSeq-a Python framework to work with high-throughput sequencing data. Bioinformatics 31, 166-169 (2015). 
69. Love, M. I., Huber, W. \& Anders, S. Moderated estimation of fold change and dispersion for RNA-seq data with DESeq2. Genome Biol. 15, 550-21 (2014).

70. Slocum, M. K. \& Parkinson, J. S. Genetics of methyl-accepting chemotaxis proteins in Escherichia coli: null phenotypes of the tar and tap genes. $J$. Bacteriol. 163, 586-594 (1985).

\section{Acknowledgements}

This research was supported by the Intramural Research Program of the NIH, NIAID. We thank Clay Ashley, Destiny Taylor, and Melissa Leath for their assistance with obtaining calves. We thank Kelly Hughes and John (Sandy) Parkinson (University of Utah) and Tino Krell (Estación Experimental del Zaidín) for helpful discussions. We thank members of our lab for critical review of the manuscript, Rose Perry for graphical assistance, Aaron Carmody for flow cytometry, and Sarah Anzick and Dan Bruno for help with RNA sequencing. This project has been funded in part from the Department of Health and Human Services under BCBB Support Services Contract

HHSN316201300006W/HHSN27200002 to MSC, Inc.

\section{Author contributions}

Conceptualization, K.G.C., A.C., L.K., B.J., T.S., and O.S.M.; Investigation, K.G.C., A.C., L.K., T.S., M.M., V.R.P., R.C.L., C.W.C., L.G.A., L.K.B., S.V.L., M.K., S.D.L.; Formal analysis, K.G.C., A.C., B.J., T.S., C.M.; Resources, C.W.C., L.G.A., L.K.B., S.D.L., O.S.M.; Software, B.J.; Writing-original draft, K.G.C. and O.S.M.; Review and editing, K.G.C., A.C., B.J., T.S., C.M., M.M., V.R.P., R.C.L., L.G.A., S.V.L., M.K., S.D.L., and O.S.M.; Visualization, K.G.C., A.C., C.M.; Supervision, O.S.M.; Funding acquisition, O.S.M.

\section{Ethics statement}

All animal studies were carried out following the recommendations in the Guide for the Care and Use of Laboratory Animals, 8th Edition (National Research Council), and the animal study protocols were approved by the Rocky Mountain Laboratories Animal Care and Use Committee (Protocol numbers 2016-035 and 2019-056). Bovine ligated ileal loop surgeries were approved by the Texas A\&M University Institutional Animal Care and Use Committee (2017-0445). Animals were euthanized before the development of clinical disease at specified time points. Human blood donations were collected from volunteers by the Department for Transfusion Medicine, NIH, with signed informed consent, acknowledging that the donation would be used for research by NIH intramural investigators. Blood collection was approved by the NIH Institutional Review Board (Protocol 99-CC-0168). Samples were maintained and used anonymously.

Competing interests

The authors declare no competing interests.

\section{Additional information}

Supplementary information is available for this paper at https://doi.org/10.1038/s41467020-20558-6.

Correspondence and requests for materials should be addressed to O.S.-M.

Peer review information Nature Communications thanks the anonymous reviewers for their contribution to the peer review of this work. Peer reviewer reports are available.

Reprints and permission information is available at http://www.nature.com/reprints

Publisher's note Springer Nature remains neutral with regard to jurisdictional claims in published maps and institutional affiliations.

(c) (i) Open Access This article is licensed under a Creative Commons Attribution 4.0 International License, which permits use, sharing, adaptation, distribution and reproduction in any medium or format, as long as you give appropriate credit to the original author(s) and the source, provide a link to the Creative Commons license, and indicate if changes were made. The images or other third party material in this article are included in the article's Creative Commons license, unless indicated otherwise in a credit line to the material. If material is not included in the article's Creative Commons license and your intended use is not permitted by statutory regulation or exceeds the permitted use, you will need to obtain permission directly from the copyright holder. To view a copy of this license, visit http://creativecommons.org/ licenses/by/4.0/.

This is a U.S. government work and not under copyright protection in the U.S.; foreign copyright protection may apply 2021 